Research Article

\title{
Impact of Stochastically Perturbed Terminal Velocities on Convective-Scale Ensemble Forecasts of Precipitation
}

\author{
Shizhang Wang $\mathbb{D}$, Xiaoshi Qiao, and Jinzhong Min \\ Collaborative Innovation Center on Forecast and Evaluation of Meteorological Disasters, \\ Key Laboratory of Meteorological Disaster of Ministry of Education, Nanjing University of Information Science and Technology, \\ Nanjing, China \\ Correspondence should be addressed to Shizhang Wang; 002568@nuist.edu.cn
}

Received 21 August 2019; Revised 17 March 2020; Accepted 1 July 2020; Published 20 July 2020

Academic Editor: Helena A. Flocas

Copyright (C) 2020 Shizhang Wang et al. This is an open access article distributed under the Creative Commons Attribution License, which permits unrestricted use, distribution, and reproduction in any medium, provided the original work is properly cited.

\begin{abstract}
The impact of stochastically perturbing the terminal velocities of hydrometeors on convective-scale ensemble forecasts of precipitation was examined. An idealized supercell storm case was first used to determine the terminal velocity error characteristics for a one-moment microphysics scheme in terms of the terminal velocities from a two-moment scheme. Two real cases were employed to evaluate the forecast skills resulting from perturbing the terminal velocities with real data. The results indicated that the one-moment scheme produced terminal velocities that were approximately three times higher than those of the twomoment scheme for snow and hail, which often resulted in overpredictions of hourly precipitation and areal accumulated precipitation. Therefore, stochastically perturbing the terminal velocities according to their error characteristics matched the observed hourly precipitation and areal accumulated precipitation better than the symmetrical perturbations. For the twomoment scheme, the symmetrical perturbations of the terminal velocities tended to produce lower falling speeds of precipitation hydrometeors; therefore, more light rain was produced. Compared to the unperturbed two-moment scheme, symmetrically perturbing the terminal velocities resulted in smaller precipitation errors when precipitation was overestimated but comparable or slightly larger precipitation errors when precipitation was underestimated. This work demonstrates the sensitivity of precipitation ensemble forecasts to terminal velocity perturbations and the potential benefits of adopting these perturbations; however, whether the perturbations actually result in significant improvements in precipitation forecast skill needs further study.
\end{abstract}

\section{Introduction}

Currently, it is widely acknowledged that numerical weather predictions should consider uncertainty estimations [1]. This uncertainty is mainly from two sources: uncertainties arising from the initial conditions and the model itself $[2,3]$. The latter has received much attention in the past decade because the former cannot explain the entire forecast uncertainty [4]. To allow for model uncertainty, there are two broadly used approaches categorized according to the number of parameterization schemes used for one physical process: (i) using a multiphysics ensemble and (ii) perturbing quantities such as the prescribed parameters or the tendencies in one parameterization scheme [3,5]. Multiphysics ensembles consist of members that use different parameterizations to estimate one physical process; the benefit to the performance of ensemble forecasts in terms of ensemble spread and forecast skill has been demonstrated in many studies (e.g., $[2,4,6-14])$. However, this approach produces different attractors among members, which causes inconsistent distributions of forecasts that are not desirable for statistical postprocessing $[5,15]$. In addition, maintaining different physics configurations requires significant resources.

The second approach avoids the deficiency of an inconsistent distribution. Perturbing quantities in one parameterization makes ensemble members different but equally likely $[16,17]$. One of these approaches is the stochastic method, which perturbs parameterizations at every time step and grid point during the integration. With this method, not only the prescribed coefficients but also the 
tendencies can be perturbed. The former is called the stochastic perturbed parameterization [18], while the latter is called the stochastic perturbation of parameterization tendency $[19,20]$. The SPPT method often perturbs the accumulated physical tendencies from the concerned parameterizations $[2,4,16,20,21]$, while the SPP method has to be designed for each parameterization (e.g., $[5,22-25])$. Both methods have been demonstrated as useful for the skill of ensemble forecasts, where the SPP is more skillful for short-range forecasts [18].

Although all parameterizations can be and have often been perturbed by the same multiplicative perturbation, separately perturbing the parameterizations has been shown to be beneficial $[3,17,24,26]$. Shutts and Pallarès [27] indicated that the error characteristics of parameterizations differ substantially. Therefore, it is not always valid to attribute the same error characteristics to all parameterizations [17]. Even in one parameterization, perturbing tendencies of different variables in the same way may not improve ensemble forecast skills [28]. Considering the different error characteristics, Sanchez et al. [26] assigned different error standard deviations for parameterizations and obtained a larger spread in the tropics. Later, Christensen et al. [3] examined the performance of independently perturbing all parameterizations and concluded that this approach improved the forecast reliability in the tropics and increased the forecast skill in the extratropics. Subsequently, Wastl et al. [17] separately perturbed parameterizations and obtained statistically significant improvements with respect to the probabilistic forecasts. These studies imply that designing the perturbation in terms of the parameterization error characteristics may benefit the forecast skill using the stochastic perturbation approach.

Among the parameterizations involved in a numerical prediction model, the present work focuses on the microphysics parameterization because it is important for convective-scale ensemble forecasting but is highly uncertain [25]. In addition to the impact of intercept parameters on storm forecasts (e.g., [29-32]), the terminal velocity of hydrometeors in precipitation forecasts is important. Parodi and Emanuel [33] revealed that the precipitation intensity increased along with the terminal velocity of rainwater, and the convective cell sizes decreased for higher terminal velocities. This relationship was confirmed by Singh and O'Gorman [34], who further demonstrated that the terminal velocity influenced the precipitation duration substantially. Bryan and Morrison [35] determined that slower terminal velocities led to a wide precipitation area. Morales et al. [36] have documented the impact of terminal velocity on orographic precipitation and revealed that the terminal velocity of snow affected the precipitation peak location. An observational study [37] concluded that the terminal velocities of wet and dry snow differed substantially, with wet snow exceeding $2 \mathrm{~m} \cdot \mathrm{s}^{-1}$ for a given diameter and dry snow reaching only $1 \mathrm{~m} \cdot \mathrm{s}^{-1}$. Moreover, wet snow is not predicted in most microphysics schemes, such as the Lin scheme [38], the WRF SM 6-class (WSM6) scheme [39], the Thompson scheme [40], the double-moment (DM) MY2 scheme [41], and the Morrison scheme [42]. In addition, terminal velocities influence the raindrop breakup process [43], but this process is not well simulated by microphysics parameterizations [44]. Moreover, the raindrop breakup process may produce super-terminal drops whose terminal velocities are higher than the model calculated terminal velocities [45]. The above studies imply that terminal velocities estimated by microphysics parameterizations may have a large error; thus, the terminal velocity uncertainty should be considered in the ensemble forecast.

To date, however, terminal velocity perturbations have not been involved in stochastic approaches. Previous studies have perturbed the intercept parameter $[22,25,28]$, the rain evaporation rate and accretion [22, 23], and the tendencies of temperature and water vapor $[4,5,15,16,21,25,28]$. Although the intercept parameter perturbation synchronously adjusts the terminal velocity, the latter is not fully determined by the former. Therefore, it is still worth examining the impact of independently perturbing the terminal velocity in convective-scale precipitation ensemble forecast with the stochastic perturbation approach, which is the main purpose of this work. In addition to examining a one-moment scheme, the terminal velocities estimated by a two-moment microphysics scheme are perturbed. Perturbing the one-moment scheme is based on the consideration that one-moment schemes have still been widely used in recent years (e.g., [46]) due to their lower computational cost. For the two-moment scheme, the large terminal velocity difference between the MY2 and Morrison schemes [32] indicates that the terminal velocity uncertainties in the two-moment schemes are still large. As a preliminary study on stochastically perturbing the terminal velocities of hydrometeors, an idealized storm case is first used to determine the error characteristics of terminal velocities for a one-moment scheme with a two-moment scheme serving as a proxy for the truth. Then, two actual precipitation cases are employed to evaluate the effects of stochastically perturbing the terminal velocities in terms of precipitation forecasting.

The remainder of this paper is organized as follows. In Section 2, the stochastic perturbation method is briefly introduced. In Section 3, the idealized and real cases are reviewed and the experimental designs are described. The results of the experiments are demonstrated and discussed in Section 4. Finally, in Section 5, the summary and conclusions are given.

\section{Methodology}

2.1. Stochastic Perturbation Method. The Advanced Regional Prediction System [47, 48] was used in this study. The abilities of this prediction model in convective-scale forecasts have been demonstrated by many studies (e.g., [49-53]). Based on this model, a stochastic perturbation method was developed by Qiao et al. [54], who followed the SPPT method of Palmer et al. [20] but generated spatial perturbations using the recursive filter proposed by Gao and Stensrud [55]. Qiao et al. [25] further developed this method by adding intercept parameter perturbations so that the method can perform the SPP for convective-scale ensemble 
forecasts. In the present work, the perturbation of terminal velocities was added. The perturbation equation is written as follows:

$$
\frac{\partial X}{\partial t}=D+\sum_{i=1}^{N-1} P_{i}+P_{\text {micro }}\left[r_{r} v t_{r}, r_{s} v t_{s}, r_{h} v t_{h}\right]
$$

where $X$ represents a prognostic variable, $N$ is the number of parameterizations, which are denoted by $P, D$ denotes the dynamic part of the forecast model, $v t$ denotes the terminal velocity, and the multiplicative perturbation is denoted by $r$, whose subscripts $r, s$, and $h$ represent the intercept parameter, rainwater, snow, and hail, respectively. The subscript "micro" is used to highlight the microphysics parameterization. Following Qiao et al. [25], all intercept parameters used the same multiplicative perturbation. Terminal velocities were separately perturbed because their error characteristics may not be identical, which will be examined later.

The perturbation resampling procedure proposed by Wang et al. [28] was adopted in this work. This procedure was designed to transform the Gaussian perturbations $(0,1)$ to an asymmetric distribution in which half of the samples have values greater than 1.0 and the other half have values less than 1.0. For instance, the perturbed intercept parameter may be 10 times smaller or 10 times larger than the prescribed value, which corresponds to a range of 0.1 to 10.0 that is not centered at 1.0. This asymmetric distribution is likely valid for terminal velocity perturbations of the onemoment scheme according to the findings of Dawson et al. [31] that the small particles may fall too quickly, which implies that there is a bias between the terminal velocity estimated by a one-moment scheme and the truth. Because this bias is currently unknown, the resampling procedure was modified in this work to generate an approximately uniform distribution of perturbation $R(r)$ within a specific range. $R(\cdot)$ is the function of perturbation $r$ which follows a Gaussian distribution. The modified procedure is as follows:

$$
R(r)= \begin{cases}\left(l_{\text {max }}-l_{\text {mid }}\right)\left(1-e^{-(r / \sigma)}\right)+l_{\text {mid }}, & r \geq 0, l_{\text {min }} \geq 0, \\ \left(l_{\text {mid }}-l_{\text {min }}\right)\left(e^{(r / \sigma)}-1\right)+l_{\text {mid }}, & r<0, l_{\text {min }} \geq 0,\end{cases}
$$

where $\sigma$ is the STD of $r, l_{\max }$ and $l_{\min }$ are the maximum and minimum of $R(r)$, respectively, and $l_{\text {mid }}$ is a value between $l_{\max }$ and $l_{\text {min }}$. For the terminal velocity perturbations, $l_{\text {mid }}$ is the average of $l_{\max }$ and $l_{\min }$. With (2), the perturbation range $\left(l_{\min }, l_{\max }\right)$ and the middle value of samples can be assigned straightforwardly. The value of $R(r)$ is used for the multiplicative perturbation $r$ in (1).

Note that the asymmetric perturbations can also be resampled in log space, which is simpler than (2); whether the stochastic approach is sensitive to the resampling perturbations needs to be examined in the future. Figure 1 shows an example of the frequency distribution of asymmetric perturbations after applying either function $R(\cdot)$ or $\log _{2}$ (using $2^{r}$ to transform). Compared to those generated by function $\log _{2}$, the frequencies generated by $R(\cdot)$ do not vary greatly (from $\sim 9.0 \%$ to $\sim 12.5 \%$ ) in $[0.6,1.6]$, which can

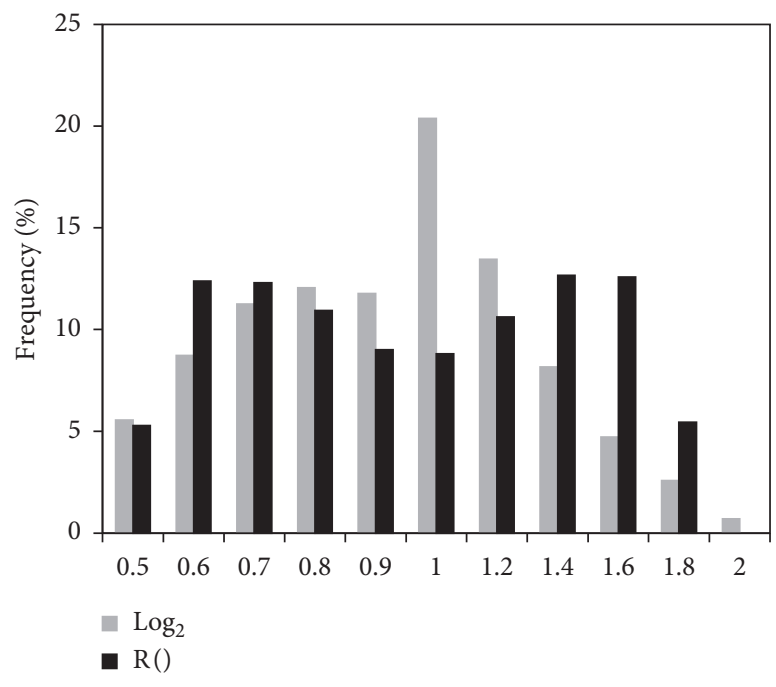

FIGURE 1: Forecast frequency distribution (\%) of the resampled samples using either function $R(\cdot)$ or function $\log _{2}$. The $l_{\text {min }}, l_{\text {mid }}$, and $l_{\max }$ are $0.5,1.0$, and 2.0, respectively. The sample size is 50000, and the initial samples followed the Gaussian distribution with a zero mean and an STD of 1.0. For function $\log _{2}$, the initial samples within \pm 1 were used.

be regarded as a quasiuniform distribution to some extent. For $R(\cdot)$, the value of 2.0 cannot be reached, so the corresponding frequency is zero.

2.2. Evaluation Metrics. As mentioned in Section 1, the forecast accuracies of stochastically perturbing the terminal velocities in an idealized case and two real cases are examined in the present work. In the idealized case, the terminal velocity frequencies of rainwater, snow, and hail as functions of mixing ratios were calculated for both the onemoment and two-moment schemes. The terminal velocity frequency difference between the one-moment and twomoment schemes was used to measure the terminal velocity bias of the one-moment scheme because the two-moment scheme is more realistic [31]. The evaluation of the precipitation ensemble forecasts was conducted in terms of the root-mean-square error (RMSE) of hourly precipitation, bias score, hourly precipitation frequency, and reliability. The ensemble spread of hourly precipitation was also examined. The RMSE and ensemble spread of hourly precipitation were calculated for ensemble means at grid points with hourly precipitations greater than $0.1 \mathrm{~mm} \cdot \mathrm{h}^{-1}$. Considering that the terminal velocity error may cause precipitation rate bias, the bias score was used to determine whether taking into account terminal velocity error benefits the accuracy of ensemble forecasts, and the precipitation frequency was employed to measure the precipitation frequency bias. The reliability of ensemble forecasts was also examined to see whether involving terminal velocity perturbations has impacts on the probability forecasts. In addition, the areal accumulated precipitation over the forecast domain [56] was calculated to measure the areal precipitation amount bias. 
To quantitatively evaluate the terminal velocity frequency difference between the one-moment and two-moment schemes, the RMSE of the terminal velocity frequencies at a given model time was calculated as follows:

(i) Sort terminal velocities of a specific hydrometeor type throughout the $3 \mathrm{D}$ domain from largest to smallest for both microphysics schemes, which yields two sorted $1 \mathrm{D}$ series $\mathbf{f}_{1}$ and $\mathbf{f}_{2}$, where $\mathbf{f}_{1}$ represents terminal velocities from the one-moment scheme and $\mathbf{f}_{2}$ denotes terminal velocities from the two-moment scheme.

(ii) Calculate the RMSE of the terminal velocity frequencies $\quad E_{f}=\sqrt{\left(1 / N_{f}\right) \sum_{i=1}^{N_{f}}\left(\alpha f_{1}(i)-f_{2}(i)\right)^{2}}$, where $N_{f}$ is the number of points with both or either $\mathbf{f}_{1}(i)$ or $\mathbf{f}_{2}(i)$ greater than zero. The term $\alpha$ is a prescribed ratio that simulates the multiplicative perturbation in a stochastic approach. A value of $\alpha$ greater than 1.0 means increasing terminal velocities, while a value smaller than 1.0 means decreasing velocities. A discussion of $\alpha$ is provided in Section 4.1.

The above steps were applied to the terminal velocities of rainwater, snow, and hail.

In the real cases, there was no terminal velocity observation available; thus, evaluations were performed for only hourly precipitation and its associated quantities. In this situation, the National Centers for Environmental Prediction (NCEP) gridded stage IV (ST4) dataset [57] served as the baseline. This dataset provides hourly precipitation with a grid spacing of $4 \mathrm{~km}$. Considering that the physical processes in the lateral boundaries differ from those in the inner domain, the verification domain was smaller than the forecast domain, as discussed in Section 3.

\section{Case Review and Experimental Design}

In this section, an idealized case and two real cases are introduced. As a preliminary study on stochastically perturbing the terminal velocities of hydrometeors, the optimal approach to perturbing these variables is unknown, especially for the one-moment scheme, which may have terminal velocity biases. Therefore, we first use the idealized case as an example case with the two-moment scheme as the truth. The real cases are used to evaluate whether the optimal stochastic perturbation determined in the idealized case benefits actual ensemble forecasts.

3.1. Idealized Case. The idealized case used in Qiao et al. [25] was adopted in this work; thus, this case is only briefly reviewed in this section. In a horizontally homogeneous environment that was generated from a modified sounding extracted on 20 May 1977 in Del City, Oklahoma [48], a supercell storm was triggered from a thermal bubble with horizontal and vertical radiuses of $10 \mathrm{~km}$ and $1.5 \mathrm{~km}$, respectively. This simulation lasted for $3 \mathrm{~h}$ within a $108 \mathrm{~km} \times 108 \mathrm{~km}$ domain at a horizontal resolution of $2 \mathrm{~km}$. There were 53 vertical levels with a minimum grid spacing of
$20 \mathrm{~m}$ near the ground and an average grid spacing of $400 \mathrm{~m}$. The simulation using the MY2 scheme served as the truth run (hereafter MY2ref), while simulations with the Lin scheme represented the situation in the presence of microphysics parameterization errors. The reason for selecting both schemes is that the rainfall prediction characteristics of both schemes have been widely studied (e.g., $[31,58])$ and the MY2 scheme is the only two-moment scheme implemented in ARPS. The simulation using the Lin scheme with a $N_{0 r}$ value of $1 \times 10^{6} \mathrm{~m}^{-4}$ and $N_{0 h}$ of $9 \times 10^{3} \mathrm{~m}^{-4}$ was regarded as the reference run (hereafter, LINref).

Ensemble forecast experiments were also conducted for the idealized case, which all used 20 members initialized from the truth state to isolate the microphysics parameterization error. A spatial decorrelation scale of $150 \mathrm{~km}$ and a temporal scale of $1 \mathrm{~h}$ were used in all idealized ensemble forecasts. LINprt_stat separately perturbed the terminal velocities of rainwater, snow, and hail in terms of statistical terminal velocity differences between LINref and MY2ref. $l_{\min }$ and $l_{\max }$ were 0.8 and 1.0 for rainwater, 0.1 and 0.5 for snow, and 0.2 and 0.7 for hail, respectively. LINprt_sym used symmetric perturbation ranging from 0.75 to 1.25 for all terminal velocities. This type of perturbation has been widely used in SPPT and SPP studies (e.g., $[4,16,21])$. Comparing LINprt_stat and LINprt_sym provides a reference to determine if it is necessary to consider the terminal velocity error characteristics. For convenience, these experiments are summarized in Table 1.

Note that the above experiments were designed with the assumption that the MY2 scheme is the truth. However, in light of the study by Morrison and Milbrandt [32], the terminal velocity uncertainty in the MY2 scheme is not ignorable. Whether representing this uncertainty benefits the forecast skill of the MY2 scheme cannot be evaluated with the idealized case; therefore, real cases have to be introduced. Additionally, with real cases, we can evaluate whether using the MY2 scheme as the baseline to perturb the terminal velocities benefits the forecast skill of the Lin scheme.

3.2. Real Cases. Two heavy precipitation cases were selected. We mainly focus on the case that occurred on 20 March 2018 in Alabama. This precipitation system produced not only tornadoes but also heavy rainfall that exceeded $30 \mathrm{~mm} \cdot \mathrm{h}^{-1}$ from $00 \mathrm{UTC}$ to 04 UTC. The associated convective cells initialized in northern Mississippi at 20 UTC on 19 March 2018 became a quasilinear convective system in the southwest-northeast direction two hours later and moved into Alabama. This convective system moved continuously eastward and reached northern Alabama at 00 UTC 20 March 2018, with the south edge being approximately $70 \mathrm{~km}$ north of Birmingham. By this time, the convective line was oriented in the west-east direction (Figure 2(a)). Over the subsequent three hours, the convective system moved southeastward and generated several tornadoes in northeastern and eastern Alabama. The precipitation caused by this convective system in Alabama ceased at approximately 05 UTC. The other case occurred on 26 May 2012 in Kansas 
TABLE 1: Configurations of the idealized case.

\begin{tabular}{|c|c|c|c|c|c|}
\hline Experiment name & Microphysics & Ensemble size & $R\left(r_{r}\right)$ & $R\left(r_{s}\right)$ & $R\left(r_{h}\right)$ \\
\hline LINref & Lin & $\mathrm{N} / \mathrm{A}$ & 1.0 & 1.0 & 1.0 \\
\hline MY2ref & MY2 & $\mathrm{N} / \mathrm{A}$ & 1.0 & 1.0 & 1.0 \\
\hline LINprt_stat & Lin & 20 & $0.8-1.0$ & $0.1-0.5$ & $0.2-0.7$ \\
\hline LINprt_sym & Lin & 20 & $0.75-1.25$ & $0.75-1.25$ & $0.75-1.25$ \\
\hline
\end{tabular}

For deterministic forecasts, the resampling function $R(\cdot)$ is forced to be the prescribed value listed in the table; for the ensemble forecasts, $l_{\min }$ and $l_{\max }$ are listed.

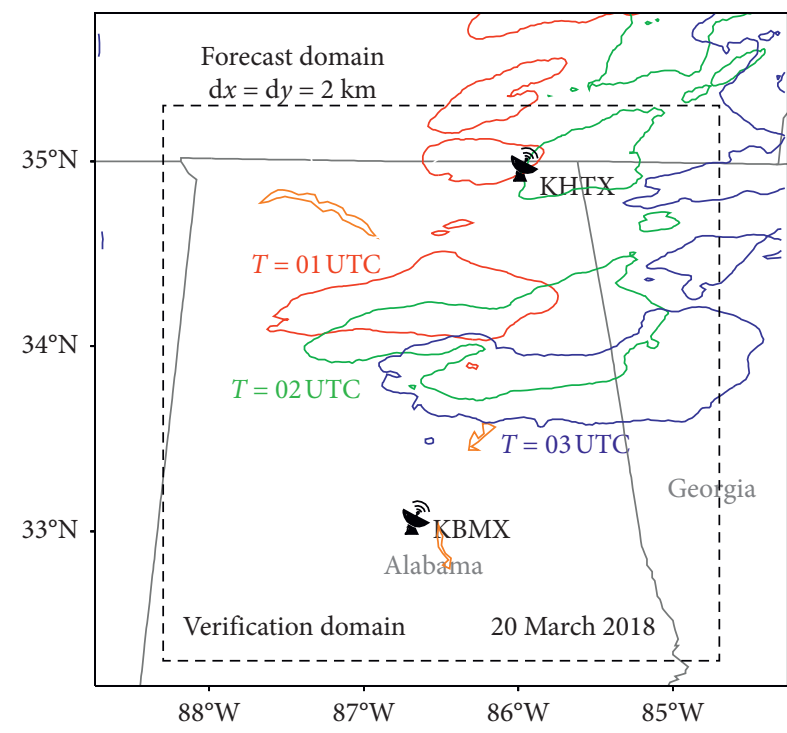

(a)

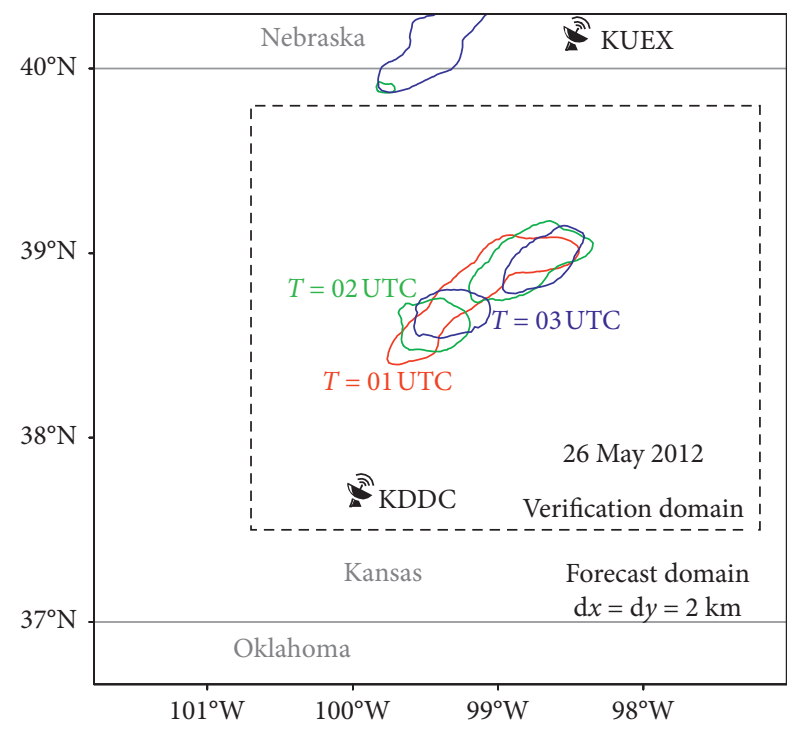

(b)

Figure 2: The forecast domains for the (a) 20 March 2018 and (b) 26 May 2012 cases, where the dashed rectangles mask the verification domains. The observed rainfall areas with precipitation greater than $5 \mathrm{~mm} \cdot \mathrm{h}^{-1}$ are plotted for $01 \mathrm{UTC}$ (red), 02 UTC (green), and 03 UTC (blue). The locations of KBMX, KHTX, KDDC, and KUEX are marked by radar icons.

and was a slow-moving supercell. The initial convective cell formed at 23 UTC 25 May 2012 approximately $80 \mathrm{~km}$ northeast of Dodge City. By 00 UTC 26 May 2012, three strong convective cells formed in a line oriented in the southwest-northeast direction. These cells stayed nearly in the same place in the first one and a half hours, producing a precipitation band oriented in the southwest-northeast direction (Figure 2(b)). By 02 UTC, there were two cells left; thus, two precipitation centers appear in Figure 2(b) (green contours). This precipitation structure (blue contours) remained by 03 UTC.

Due to computational limitations, small forecast domains were used, which also restricted the forecast lead time. In this circumstance, the present work focused on the shortterm precipitation forecast from 00 UTC 20 March 2018 to 03 UTC 20 March 2018 and from 00 UTC 26 May 2012 to 03 UTC 26 May 2012. Our results in Section 4.1 indicate that the impacts of the stochastic perturbations of the terminal velocities on precipitation take effect within $3 \mathrm{~h}$, and thus a $3 \mathrm{~h}$ forecast is assumed to be sufficient to study the above impact in the real cases, especially when precipitation has occurred. The heavy precipitation areas were approximately located at the centers of the forecast domains (Figure 2). For both cases, the forecast domain size was $406 \mathrm{~km} \times 406 \mathrm{~km}$ at a horizontal resolution of $2 \mathrm{~km}$. The number of vertical levels was 43 with the highest resolution of $20 \mathrm{~m}$ near the surface and an average resolution of $500 \mathrm{~m}$. The model top is at approximately $20 \mathrm{~km}$ above ground level (AGL). Twenty initial ensemble members at 00 UTC were generated by the approach used by Wang et al. [28]. In this approach, the three-dimensional variational (3DVar) data assimilation (DA) system [59] was run in parallel using the Global Forecasting System (GFS) analysis data at a horizontal resolution of $0.5^{\circ}$ and the perturbed radar data from KBMX, KHTX, KDDC, and KUEX, the locations of which are marked by radar icons in Figure 2. All settings, including both the 3DVar and smooth random perturbation steps for generating the initial members, were identical to those of Wang et al. [28], whose model configurations were also used in this work except for those involved in the stochastic perturbation. The model parameterization configurations are briefly discussed here. The long and shortwave radiation schemes based on Chou [60] and Chou and Suarez [61], respectively, were adopted. The planetary boundary layer scheme proposed by Sun and Chang [62] was employed. A 1.5-order turbulence kinetic energy (TKE) scheme [63] was used for the subgrid-scale turbulence parameterization. All parameterizations used the default values in ARPS. Four 
ensemble forecast experiments were designed, and all of them adopted the two-step generated initial members. LINens and MY2ens used the Lin scheme and MY2 scheme, respectively, and employed no stochastic perturbation. LINprt_ref used the same $l_{\min }$ and $l_{\max }$ as LINprt_stat. MY2prt_ref used the symmetric perturbation with a maximum amplitude of 0.25 . The terminal velocity of graupel in MY2prt was not perturbed. These perturbations were selected because the terminal velocity biases of the two-moment scheme are unknown at this time. The spatiotemporal decorrelation scales were $100 \mathrm{~km}$ and $1 \mathrm{~h}$. The above ensembles were applied to the Alabama and Kansas cases, and the suffix $\mathrm{K}$ was added to the experiment names (namely, LINens_K, MY2ens_K, LINprt_ref_K, and MY2prt_ref_K) of the Kansas case. The above experiments are listed in Table 2 except for those designed for the Kansas case.

\section{Results}

In this section, the results are examined. We first focus on the idealized case so that the optimal approach to perturb the terminal velocity from the one-moment scheme can be determined. Moreover, the impacts of increasing and decreasing the terminal velocities on the precipitation structure and amount can be determined for the one-moment scheme. For the real cases, we focus on the Alabama case because the observed precipitation distribution was well captured by almost all the experiments, thus allowing us to investigate the impact of perturbing the terminal velocities on precipitation amounts. The results of the Kansas case are briefly examined to distinguish the case-dependent impacts of the terminal velocity perturbations.

\subsection{Idealized Case}

4.1.1. The Relationship between Precipitation and Terminal Velocity. The hourly precipitation produced by the Lin scheme and MY2 scheme in the idealized case is first examined. The main difference between the precipitation forecasts of the two schemes is that LINref produced a maximum hourly precipitation exceeding $180 \mathrm{~mm} \cdot \mathrm{h}^{-1}$, while the corresponding value in MY2ref was approximately $50 \mathrm{~mm} \cdot \mathrm{h}^{-1}$. To determine the cause of this difference, the vertical mixing ratio profiles across the updraft maximum are investigated (Figure 3). The distance between the peak of falling hail (area B) and the updraft core (area A) is larger in MY2ref than in LINref. Considering that both experiments use the same environment, it is likely the slower falling speed of hail in MY2ref that allows the hail to be transported to a more distant location, which is similar to the findings of Morales et al. [36], which demonstrated that a slower falling speed resulted in more snow being transported to the downwind area of the mountain top. In addition, due to the faster falling speed, more hail (with respect to $0.1 \mathrm{~g} \cdot \mathrm{kg}^{-1}$ contour beneath the updraft core) reaches the ground in LINref, which is also the likely cause of the larger hourly precipitation maximum in LINref because melting hail is an important source of rainwater [38]. Additionally, the cold pool is stronger in LINref. Previous studies [31, 64] have stated that one-moment schemes tend to produce more small hydrometeors, which intensifies the evaporation effect and causes a stronger cold pool. The results in Figure 3 imply that the strong melting effect of hail also contributes to the strong cold pool in LINref.

Because terminal velocities may strongly influence precipitation forecasts, the distributions of terminal velocities with respect to the hydrometeor mixing ratios are examined. Figure 4 shows the corresponding distribution at the model time of $2 \mathrm{~h}$. For rainwater (Figure 4(a)), there is no clear bias between the one-moment and two-moment schemes. Moreover, the terminal velocities in MY2ref reach $10 \mathrm{~m} \cdot \mathrm{s}^{-1}$ even when $q_{r}$ is small, which is the likely result of more large raindrops being produced in MY2ref, leading to a larger mass-weighted terminal velocity [31, 41]. A further increase in $q_{r}$ has very little impact on the maximum terminal velocity in MY2ref but is accompanied by a monotonous terminal velocity increase in LINref. The terminal velocity differences between LINref and MY2ref for snow and hail are more pronounced (Figures 4(b) and 4(c)), as the terminal velocities in LINref are often three times larger than their counterparts in MY2ref. In terms of this result, the presence of higher terminal velocities in LINref than in MY2ref is confirmed.

To determine if the terminal velocity differences are caused by precipitation differences, the terminal velocities were tuned by varying $\alpha$ (mentioned in Section 2.2) from 0.1 to 1.0. First, it is necessary to determine the optimal $\alpha$ to minimize the terminal velocity difference. Because the terminal velocities differ substantially, the normalized $E_{f}$ is shown in Figure 4(d). The results in Figure 4(d) indicate that further reducing the terminal velocity of rainwater causes a larger terminal velocity difference between LINref and MY2ref; thus, the optimal $\alpha$ should be close to 1.0. The optimal values of $\alpha$ are approximately 0.3 and 0.4 for snow and hail, respectively, which is consistent with the results in Figures 4(b) and 4(c), where the terminal velocities in LINref are often larger. The terminal velocity distributions and the normalized error curve differ at different model times, although the distributions are qualitatively similar (not shown).

4.1.2. Ensemble Forecasts. First, a quantitative analysis is conducted to evaluate the forecast skills of ensemble forecasts with the two-moment scheme as the reference. Figure 5 shows the RMSE and ensemble spread for the ensemble forecast experiments. With respect to the ensemble mean RMSE, all ensemble forecasts outperform the deterministic forecast using the unperturbed Lin scheme (Figure 5(a)), which demonstrates the benefit of using ensemble forecasts. Compared with other ensemble forecast experiments, LINprt_stat produces the smallest hourly precipitation RMSE (Figure 5(a)), difference between the RMSE and ensemble spread). For clarity, the bias scores in Figure 5(b) are shown as logarithmic values, where a value of zero means no bias. Both experiments used the Lin scheme under forecasted hourly precipitation less than $5 \mathrm{~mm} \cdot \mathrm{h}^{-1}$, but LINprt_stat generated a smaller bias, consistent with results 
TABLE 2: Configurations for the Alabama case.

\begin{tabular}{lcccccc}
\hline Experiment name & Microphysics & $R\left(r_{\mathrm{r}}\right)$ & $R\left(r_{s}\right)$ & $R\left(r_{h}\right)$ & Spatial scale $(\mathrm{km})$ & Temporal scale $(h)$ \\
\hline LINens & Lin & 1.0 & 1.0 & 1.0 & N/A & N/A \\
MY2ens & MY2 & 1.0 & 1.0 & 1.0 & N/A & N/A \\
LINprt_ref & Lin & $0.8-1.0$ & $0.1-0.5$ & $0.2-0.7$ & 100 & 1 \\
MY2prt_ref & MY2 & $0.75-1.25$ & $0.75-1.25$ & $0.75-1.25$ & 100 & 1 \\
\hline
\end{tabular}

For the resampling function $R(\cdot), l_{\min }$ and $l_{\max }$ are listed. In LINens and MY2ens, the value of $R(\cdot)$ is forced to be a constant of 1.0 so that terminal velocities are not perturbed.

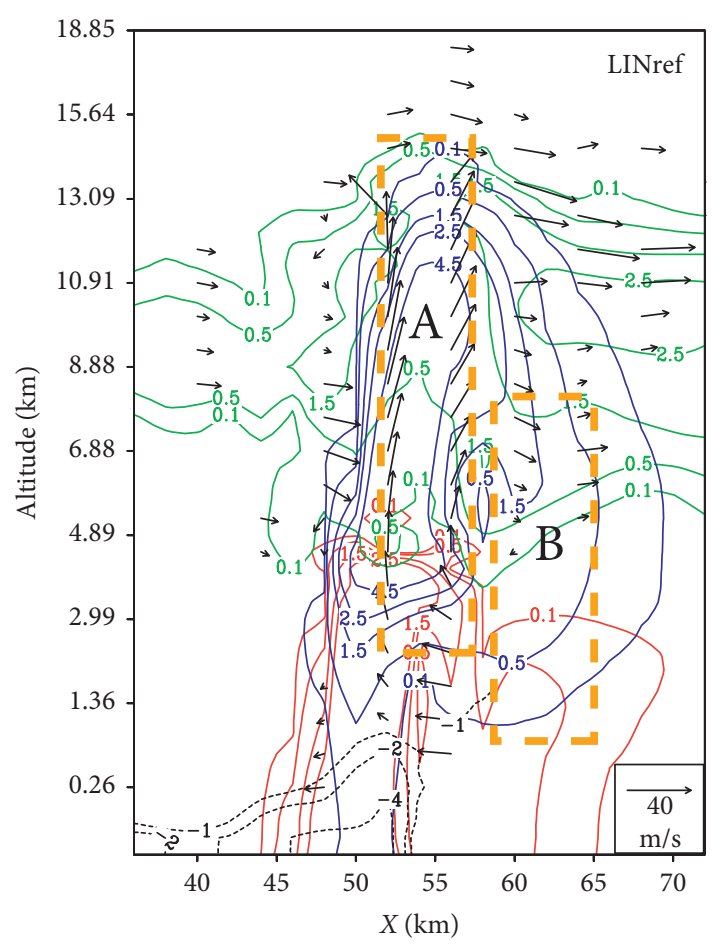

(a)

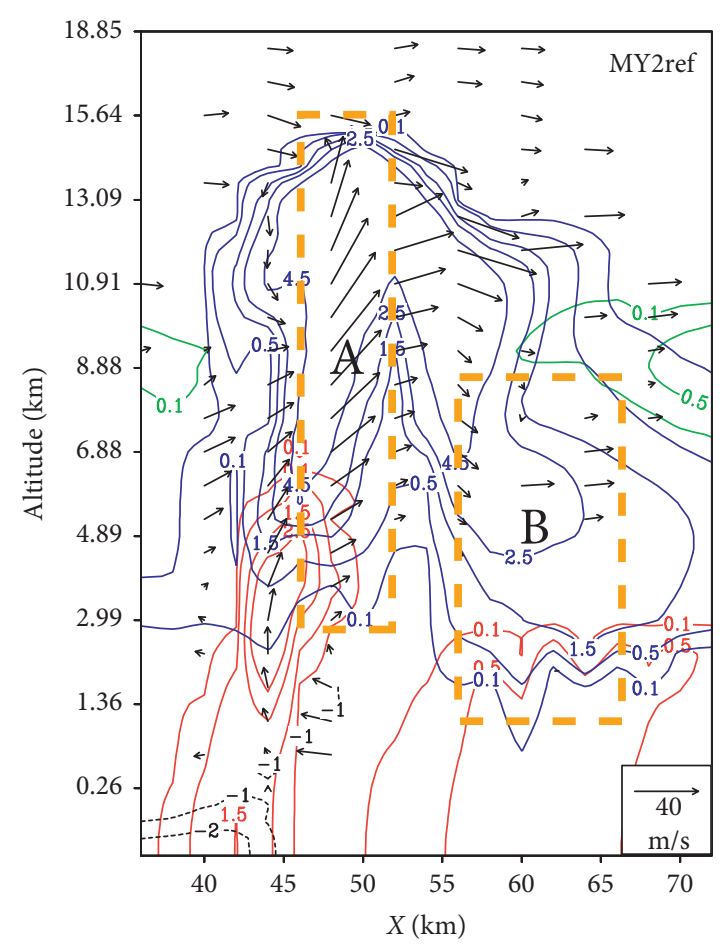

(b)

FiguRE 3: Vertical cross-sections of $q_{r}$ (red), $q_{s}$ (green), $q_{h}$ (blue), perturbation potential temperature (black contour), and wind (vector, for the absolute vertical velocity greater than $1.0 \mathrm{~m} \cdot \mathrm{s}^{-1}$ ) at $T=2 \mathrm{~h}$ in the $X-Z$ plane along (a) $y=60 \mathrm{~km}$ and (b) $y=58 \mathrm{~km}$ for (a) LINref and (b) MY2ref, respectively. Both $X-Z$ planes cross the maximum updraft centers in supercell. Area A denotes the updraft core; area B denotes the hail falling outside the updraft core.

in Figure 5(c), which show that LINprt_stat produced an hourly precipitation frequency close to the true value. For hourly precipitation greater than $10 \mathrm{~mm} \cdot \mathrm{h}^{-1}$, LINprt_stat yielded the largest bias, but this bias becomes pronouncedly smaller when the hourly precipitation is over $30 \mathrm{~mm} \cdot \mathrm{h}^{-1}$. In contrast, the bias scores of LINprt_sym are close to those of LINref for all examined thresholds, indicating that symmetric perturbations cannot represent the terminal velocity error when systematic bias exists (the terminal velocities of snow and hail in the Lin scheme overestimate the values in the MY2 scheme) and implies that there is a benefit to perturbing a parameterization in terms of its error characteristics.

The better estimated precipitation frequency and smaller bias allow LINprt_stat to better predict the areal accumulated precipitation of MY2ref than the LINprt_sym members throughout the forecast (Figure 5(d)), which indicates that the smaller hourly precipitation RMSE obtained by LINprt_stat is mainly obtained through a better precipitation amount forecast. LINref produces an areal accumulated precipitation value that is much larger than that of MY2ref, which is mainly attributable to the excessively large precipitation maximum $\left(180 \mathrm{~mm} \cdot \mathrm{h}^{-1}\right.$ in LINref and $50 \mathrm{~mm} \cdot \mathrm{h}^{-1}$ in MY2ref). The similar mean areal accumulated precipitation values of LINprt_sym and LINref are expected because the terminal velocity perturbations are symmetric so that the overpredicted precipitation in some members is offset by other members that predict less rainfall with terminal velocity perturbations smaller than 1.0.

In addition to precipitation amount forecasts, the precipitation probability forecasts were also examined. For forecast probabilities lower than 0.3 , LINprt_stat is more reliable than LINprt_sym for thresholds from $5 \mathrm{~mm} \cdot \mathrm{h}^{-1}$ to $20 \mathrm{~mm} \cdot \mathrm{h}^{-1}$. This difference between the two experiments is also valid for forecast probabilities over 0.7. For forecast probabilities between 0.3 and 0.7 , the reliabilities of both experiments do not substantially differ, except for the threshold of $20 \mathrm{~mm} \cdot \mathrm{h}^{-1}$, where LINprt_stat is consistently 


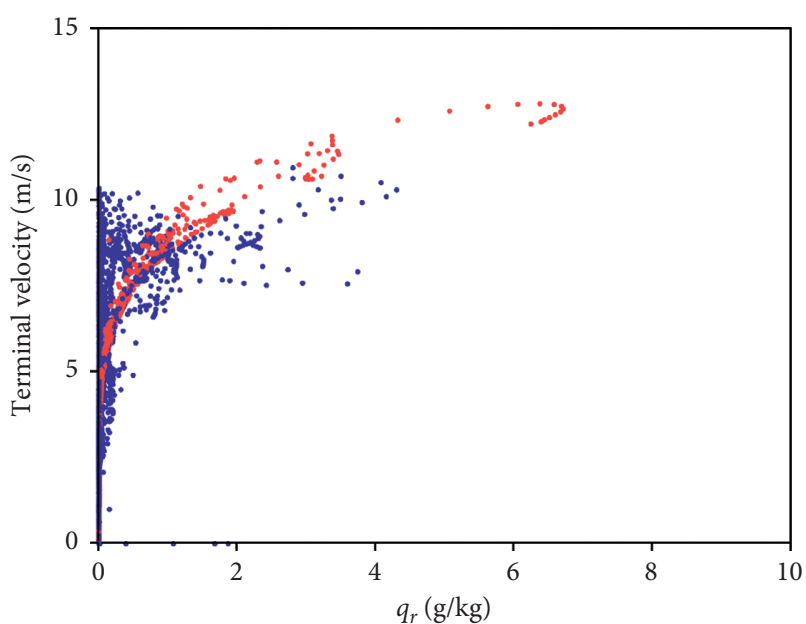

(a)

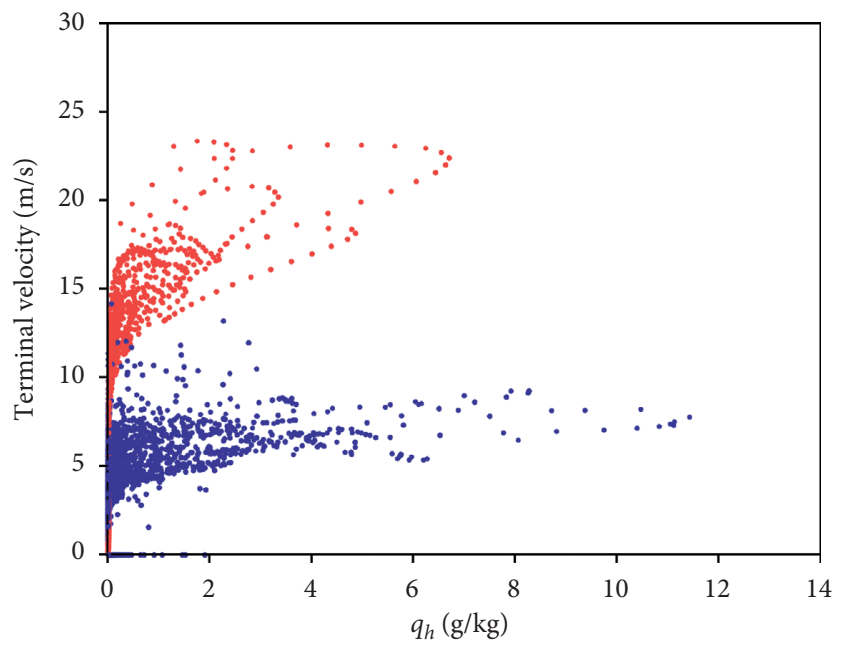

(c)

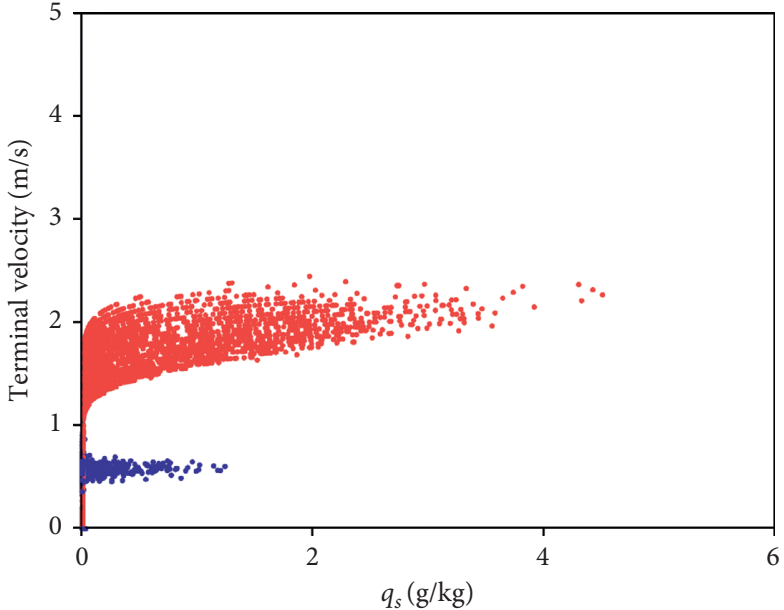

(b)

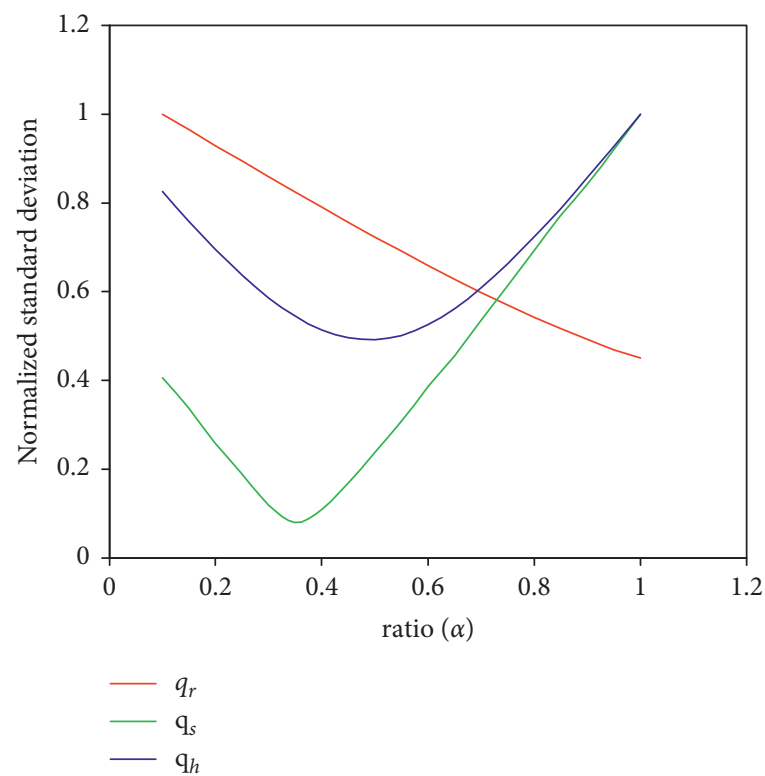

(d)

Figure 4: Terminal velocity distributions with respect to (a) $q_{r}$, (b) $q_{s}$, and (c) $q_{h}$ at $T=2 \mathrm{~h}$ for all model levels. Terminal velocities yielded by LINref are shown with red dots and those of MY2ref are blue. The normalized standard deviations between the terminal velocity frequencies of LINref and MY2ref are shown in (d) for rain (red), snow (green), and hail (blue).

better than LINprt_sym. The reason is that most of the LINprt_stat members produce a spatial precipitation distribution similar to that of MY2ref, so the high probability in this experiment is generally consistent with the truth. In contrast, LINprt_sym overestimates the probability of heavy rain $\left(>20 \mathrm{~mm} \cdot \mathrm{h}^{-1}\right)$ because LINprt_sym has some members with substantially high terminal velocity values (due to symmetric perturbations); consequently, the areas with hourly precipitation greater than $20 \mathrm{~mm} \cdot \mathrm{h}^{-1}$ are small and not well collocated with the truth.

Although terminal velocity perturbations have yielded potential benefits in precipitation ensemble forecasts, the above results are determined against the two-moment scheme, which may not be the best scheme in some cases (e.g., $[65,66])$. Therefore, it is necessary to examine the forecast skills using these perturbation approaches in real cases for both one-moment and two-moment schemes, as the evaluation of the stochastic perturbation of the twomoment scheme can only be done with real cases.

\subsection{Real Cases}

4.2.1. Alabama Case. Figure 6(a) shows the hourly precipitation error of the ensemble mean and ensemble spread. The results in this figure indicate that perturbing the terminal velocities can reduce precipitation errors regardless of the microphysics scheme. Intuitively, terminal velocity perturbations have a larger impact on the one-moment scheme than that on the two-moment scheme. However, perturbing the terminal velocities does not increase precipitation forecast spread, as the ensemble spread in all 


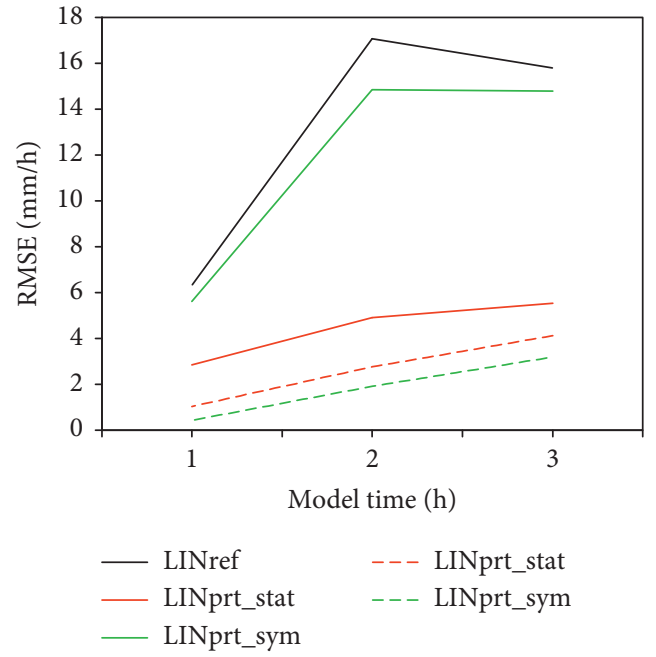

(a)

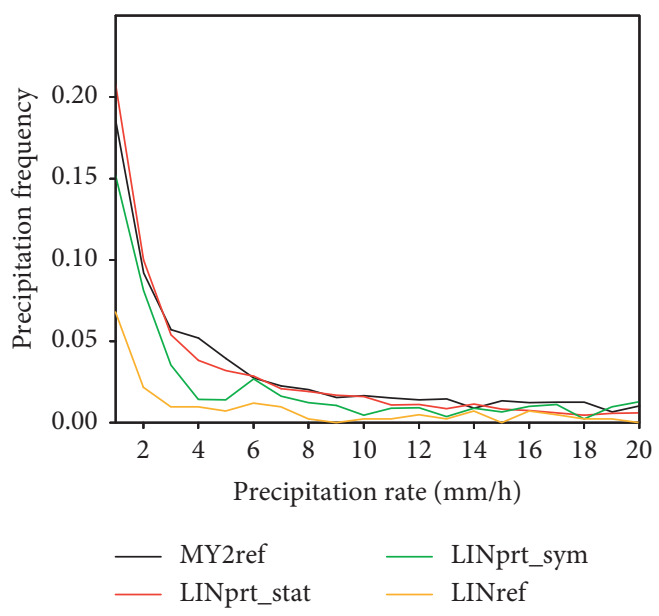

(c)

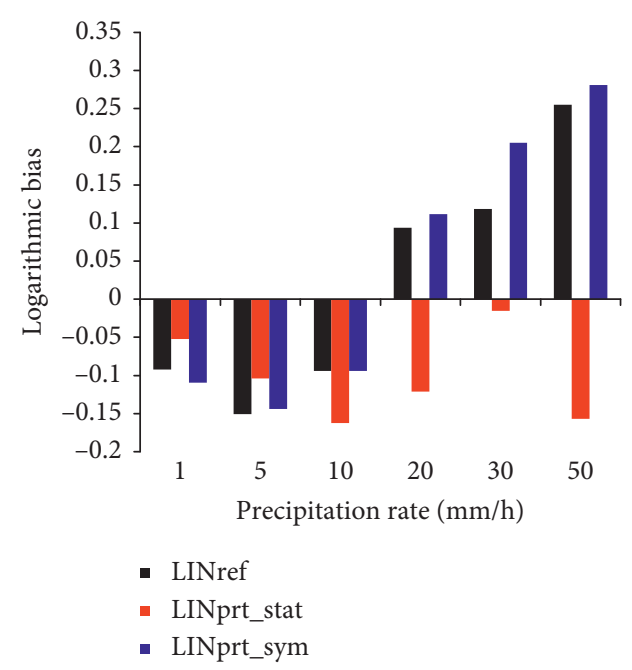

(b)

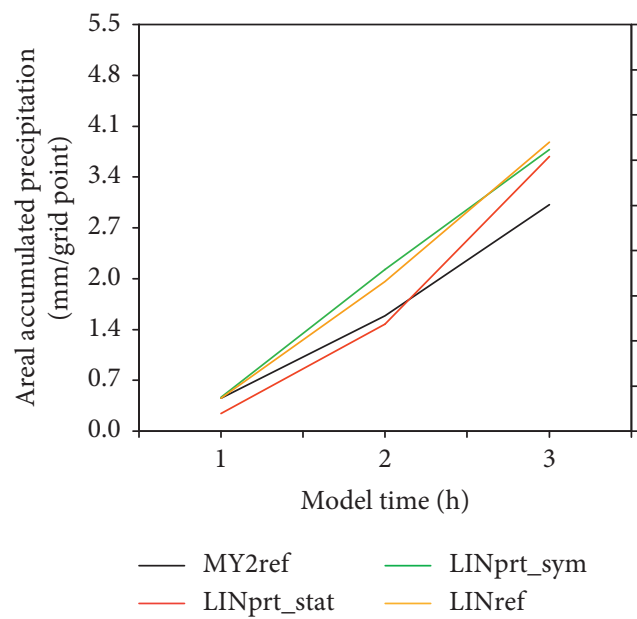

(d)

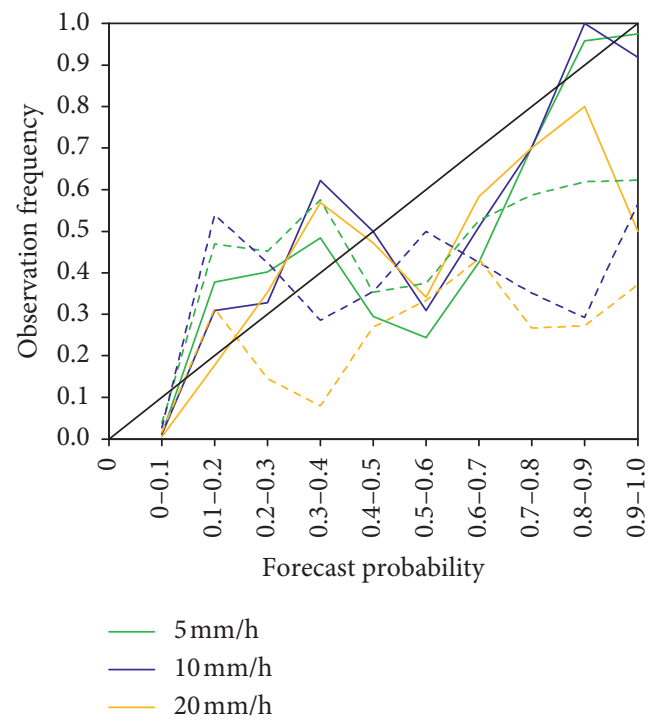

(e)

FIGURE 5: (a) RMSEs of the ensemble mean (solid) and ensemble spread (dashed) for hourly precipitation, (b) the logarithmic bias of hourly precipitation averaged over all members and all times, (c) the time averaged hourly precipitation frequency throughout the entire ensemble, (d) the areal accumulated hourly precipitation where the values for LINprt_stat and LINprt_sym are averaged over all members, and (e) the reliability diagram for LINprt_stat (solid) and LINprt_sym (dashed). The MY2 scheme serves as the truth. 


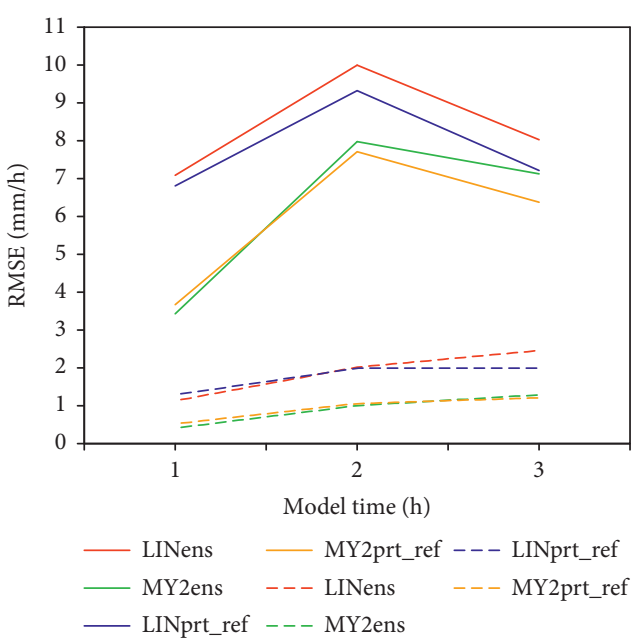

(a)

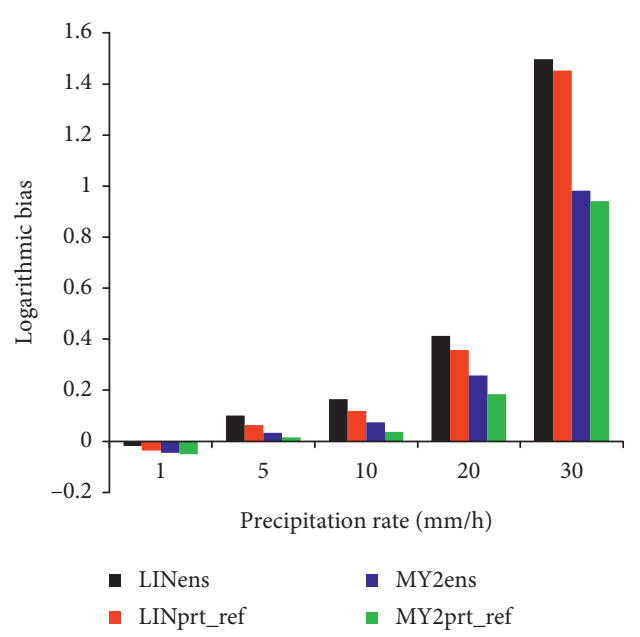

(c)

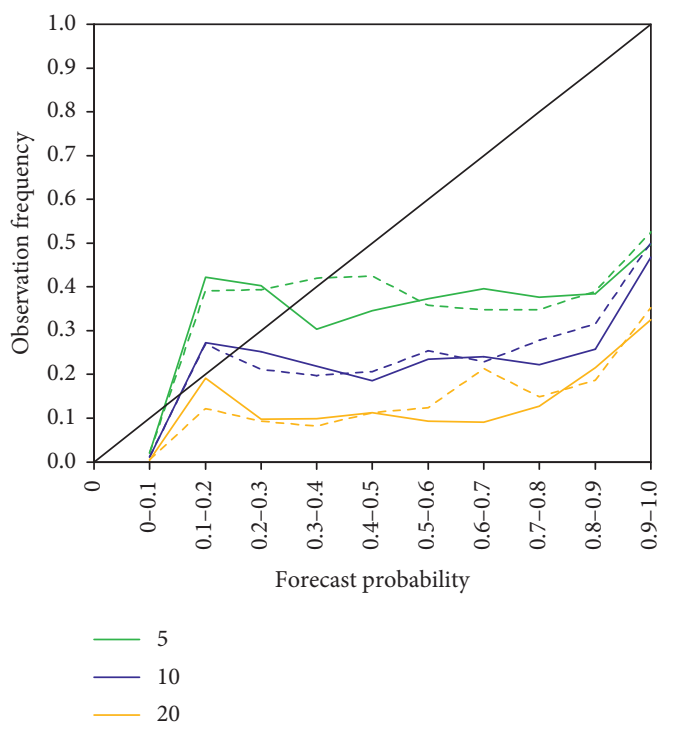

(e)

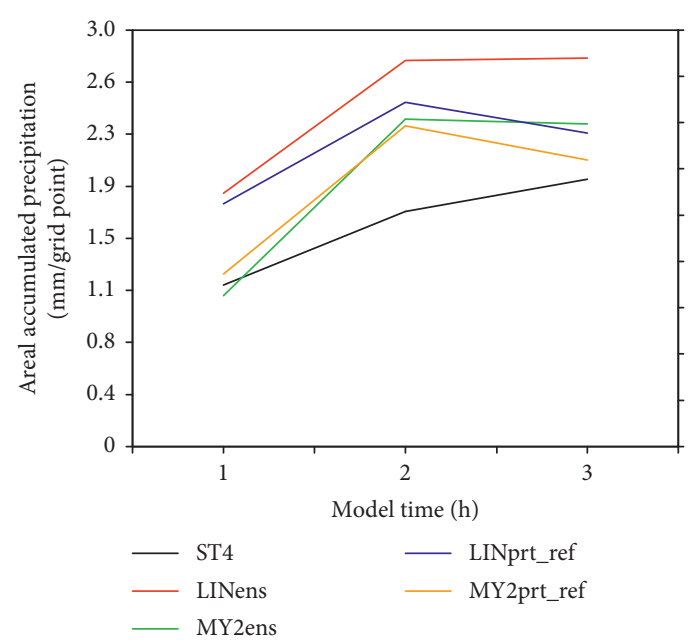

(b)

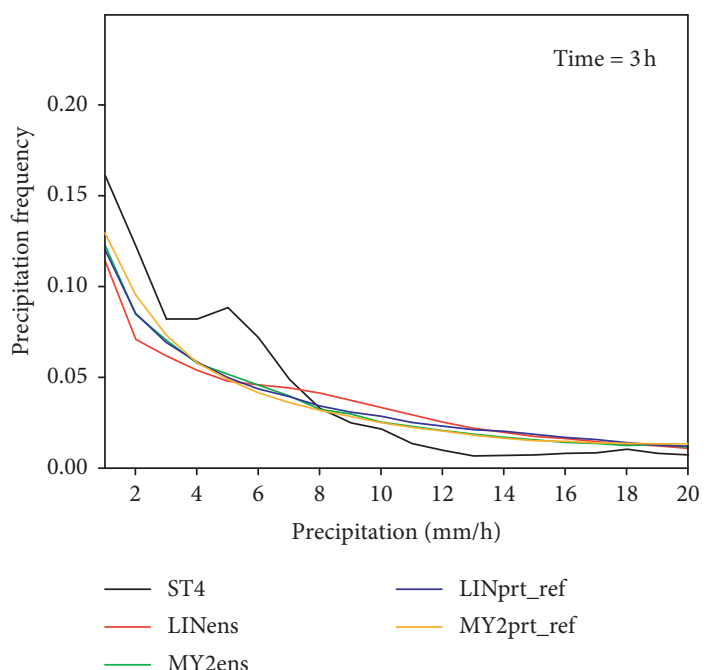

(d)

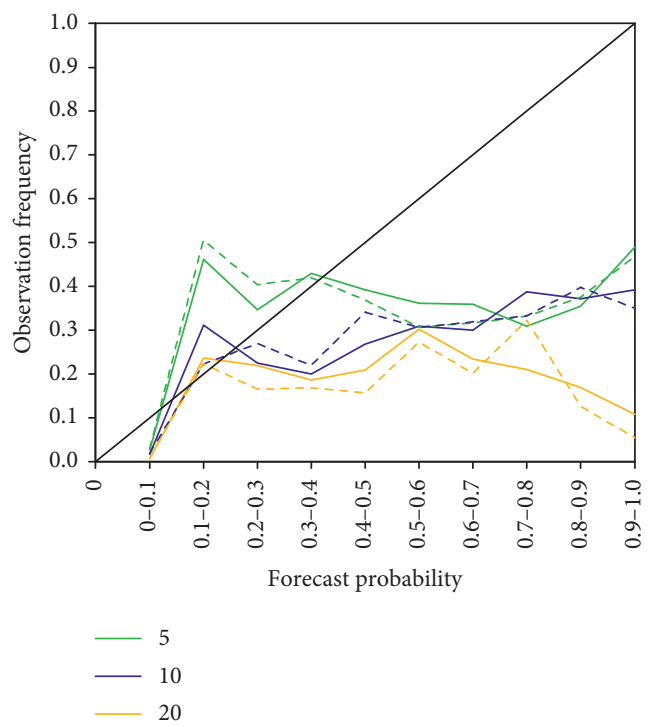

(f)

FIGURE 6: (a) RMSEs of the ensemble mean (solid) and ensemble spread (dashed) of hourly precipitation in the Alabama case, (b) averaged areal accumulated precipitation of ensemble members and observations, (c) the logarithmic bias of hourly precipitation averaged over all ensemble members and times, (d) the observed precipitation frequency (black) and the corresponding ensemble frequencies at 03 UTC, the reliability diagrams for (e) LINens (solid) and LINprt_ref (dashed) and (f) MY2ens (solid) and MY2prt_ref (dashed). 
experiments underestimates the hourly precipitation RMSE. A plausible cause is that terminal velocity perturbations are effective only in areas with nonzero hydrometeors, and the difference among members was zero in the initial members to prevent the introduction of spurious precipitation [28]. Using a more advanced method to generate initial members may increase the ensemble spread, although this is beyond the scope of the present work. In addition, the results in Figure 6(a) also indicate that the MY2 scheme outperforms the Lin scheme even when the terminal velocity perturbations are applied to the Lin scheme. Although MY2prt_ref requires much more computational resources than LINprt_ref, a two-moment scheme is preferable when the computational resources are sufficient.

The results of areal accumulated precipitation (Figure 6(b)) indicate that all experiments overestimated the precipitation amount in the verification domain, even when the two-moment scheme was adopted. Despite the above bias, the experiments with terminal velocity perturbations produced areal accumulated precipitation values closer to the observation than the experiments with only initial conditions, especially in the last two hours. Further examination (not shown) indicates that most members of LINprt_ref and MY2prt_ref yielded lower areal accumulated precipitation values than their counterparts in LINens and MY2ens, implying that the better forecasts of the areal accumulated precipitation are unlikely to have occurred by chance.

The bias scores (Figure 6(c)) demonstrate that all experiments have small biases at the light rain $\left(1 \mathrm{~mm} \cdot \mathrm{h}^{-1}\right)$ threshold but larger biases as the threshold increases. All experiments overpredicted the precipitation events at thresholds over $5 \mathrm{~mm} \cdot \mathrm{h}^{-1}$, indicating that the areal accumulated precipitation errors are mainly attributable to the biases associated with moderate and heavy rain. Even though large biases exist, LINprt_ref and MY2prt_ref produced smaller biases than LINens and MY2ens, except for light rain, indicating that perturbing the terminal velocity is likely to benefit the precipitation forecasts in real cases. The examination of precipitation frequencies (Figure 6(d)) further confirms the above improvement in precipitation amounts. LINprt_ref produced a precipitation frequency comparable to that of MY2ens for precipitation rates lower than $10 \mathrm{~mm} \cdot \mathrm{h}^{-1}$, which can be partly explained by the perturbation parameters in LINprt_ref being tuned according to the difference between the Lin scheme and the MY2 scheme. MY2prt_ref yielded a precipitation frequency closest to the observation, and thus it has the smallest RMSE and precipitation bias.

The good performance of LINprt_ref is attributable to the terminal velocity perturbations being systematically set to values smaller than 1.0, but the reason for the good performance of MY2prt_ref is not that straightforward because symmetric perturbations were used. Considering that the MY2 scheme allows a raindrop breakup effect, a plausible explanation is that perturbation greater than 1.0 increases not only the terminal velocities of hydrometeors but also the collisional kinetic energy (CKE), which increases the chance of raindrop breakup [43, 67]. As raindrop breakup occurs, the number of smaller raindrops increases, which decreases the mass-weighted terminal velocity so that the amount of rainwater reaching the ground does not increase as much as when using the Lin scheme. Further investigation (not shown) indicates that more small raindrops appear when the precipitation area is collocated with perturbations greater than 1.0, supporting the above speculation.

Although terminal velocity perturbations have positive impacts on precipitation amount forecasts, the impact on precipitation probability forecasts is not so clear. The reliability results (Figures 6(e)-6(f)) indicate that all experiments overestimated the precipitation probability. There is no significant difference between experiments with and without terminal velocity perturbations. Further examination (not shown) indicates that the overestimation is associated with the fact that the predicted precipitation areas in all experiments were larger than the observed precipitation areas, consistent with the overprediction shown in Figure 6(c).

Figure 7 shows an intuitive comparison of the best members in LINens, MY2ens, LINprt_ref, and MY2prt_ref with respect to the hourly precipitation RMSE. All of these members capture the observed precipitation band but differ in detail. At 01 UTC, members of LINens and LINprt_ref overpredicted the observed precipitation maximum, which is better matched by members of MY2ens and MY2prt_ref. This difference is consistent with Figure 6(b), in which the observed areal accumulated precipitation is well matched with MY2ens and MY2prt_ref. At 02 UTC, all members in Figure 7 overpredict the precipitation amount in northeastern Alabama, which corresponds to the increased error in all experiments in Figure 6. At 03 UTC, the observed precipitation peak $\left(>30 \mathrm{~mm} \cdot \mathrm{h}^{-1}\right)$ is approximately captured by all members, where the LINens and LINprt_ref members are intuitively better than the MY2ens and MY2prt_ref members in terms of the precipitation peak distribution. To the northeast of this observed maximum, a pronounced overprediction occurs in LINens, while the overprediction was alleviated in LINprt_ref, consistent with the results in Figures 6(b) and 6(c).

To determine the cause of the overprediction in northwestern Georgia (along approximately $34.4^{\circ} \mathrm{N}$ ) in LINens member 2, the vertical distributions of hydrometeors and their terminal velocities are examined (Figure 8). Along $34.4^{\circ} \mathrm{N}$, LINens member 2 has the smallest meridional average $q_{h}$ in the mid-levels. However, this member produces the largest falling speeds of both hail and rainwater among the examined members. Therefore, the overestimated terminal velocities result in overpredicted precipitation in LINens member 2. Otherwise, the smaller $q_{h}$ in this member should have produced smaller precipitation amounts with terminal velocities identical to that of LINprt_ref member 2 . In addition, the rainwater terminal velocity in LINprt_ref member 2 is also smaller than that of MY2ens member 2 in northwestern Georgia, which leads to the precipitation intensity in LINprt_ref member 2 being closer to the observation. This situation is also valid for the comparison between MY2prt_ref and MY2ens. The above results imply 


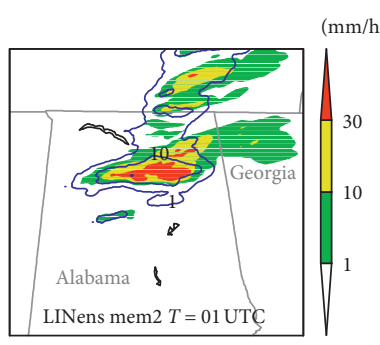

(a)

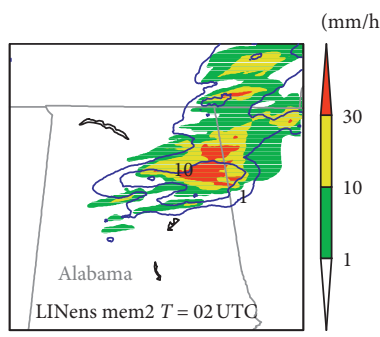

(e)

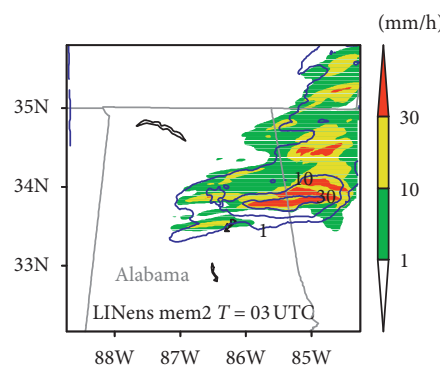

(i)

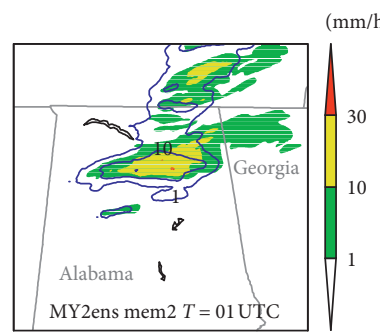

(b)

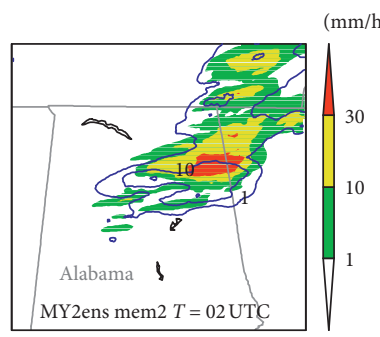

(f)

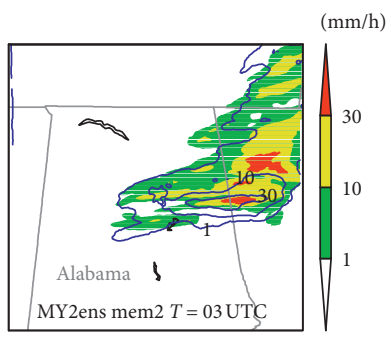

(j)

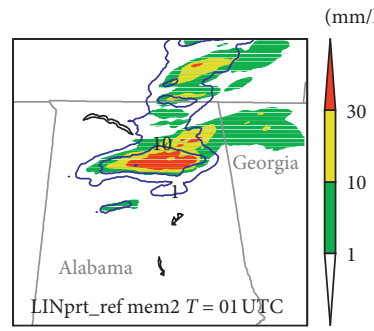

(c)

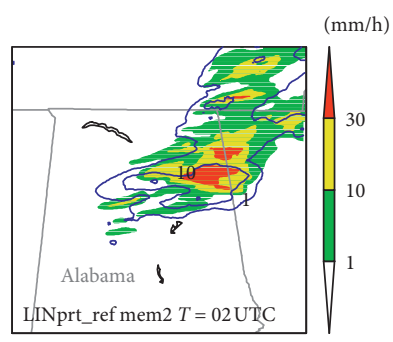

(g)

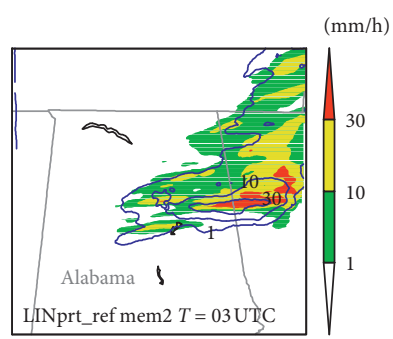

$(\mathrm{k})$

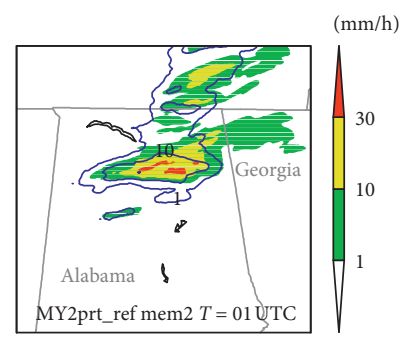

(d)

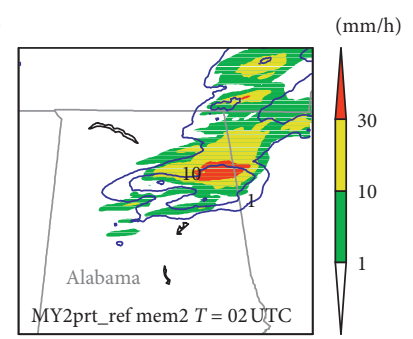

(h)

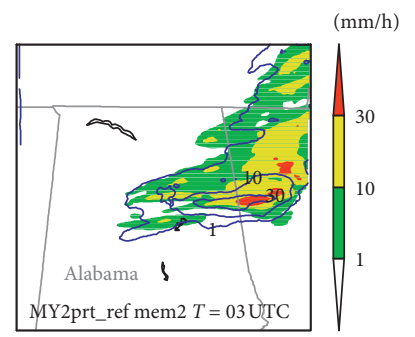

(1)

FIgURe 7: Hourly precipitation rate from the observations (contour) and (a, e, i) LINens, (b, f, j) MY2ens, (c, g, k) LINprt_ref, and (d, h, l) MY2prt_ref at 01 ZUTC (upper panels), 02 ZUTC (middle panels), and 03 ZUTC (lower panels).

that the terminal velocities yielded by a two-moment scheme are not necessarily perfect; thus, the stochastic perturbation approach applied to hydrometeor terminal velocities could aid in the skill of precipitation forecasting. However, notably, the overpredicted precipitation in MY2ens does not have to result from inaccurate terminal velocities; the contents of hail and rain may be overestimated, which can also cause a large amount of precipitation.

4.2.2. Kansas Case. The benefits from stochastically perturbing the terminal velocities yielded by the Lin scheme are again found in the Kansas case in terms of the RMSE of hourly precipitation (Figure 9(a)) and the areal accumulated precipitation (Figure 9(b)). Additionally, the ensemble spread is still insensitive to the terminal velocity perturbations. In this case, the overprediction of areal accumulated precipitation occurs in all experiments; thus, most members in LINprt_ref_K, which allows a terminal velocity bias, produce areal accumulated precipitation values that are close to the observations, compared to that of LINens_K. The similar trends in the precipitation error and areal precipitation amount in LINprt_ref_K and LINens_K (Figure 9(a) and 9(b)) imply that the smaller precipitation error is mainly attributable to the better prediction of the areal precipitation amount in LINprt_ref_K.
The better precipitation amount forecast in LINprt_ref_K corresponds to a smaller bias (Figure 9(c)) and a precipitation frequency that is close to the observations; an example at 03 UTC is shown in Figure 9(d). Unlike LINprt_ref_K, MY2prt_ref_K does not outperform MY2ens_K in terms of the precipitation error (Figure 9(a)) and the areal accumulated precipitation (Figure 9(b)). However, MY2prt_ref_K still produces a smaller bias than MY2ens_K, indicating that the impact of terminal velocity perturbations on the MY2 scheme is similar in both cases, although the precipitation forecast skill with the terminal velocity perturbations depends on the case. The reliabilities of the probability forecasts in the Kansas case (not shown) are low in all cases, indicating again that terminal velocity perturbations mainly contribute to improvements in the predicted precipitation amount. To determine the plausible causes of the consistent outperformance of perturbing terminal velocities in the Lin scheme and the case-dependent performance of terminal velocity perturbations in the MY2 scheme, we qualitatively examined the precipitation structure in all experiments for the Kansas case.

For each ensemble, the member having the smallest precipitation RMSE in the ensemble at 03 UTC was selected for the qualitative analysis. In Figure 10, all precipitation 


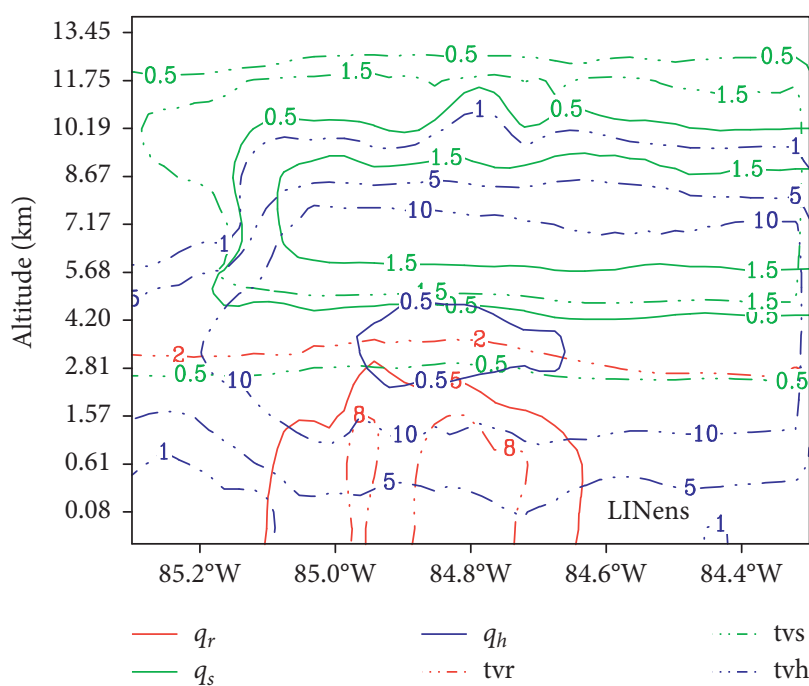

(a)

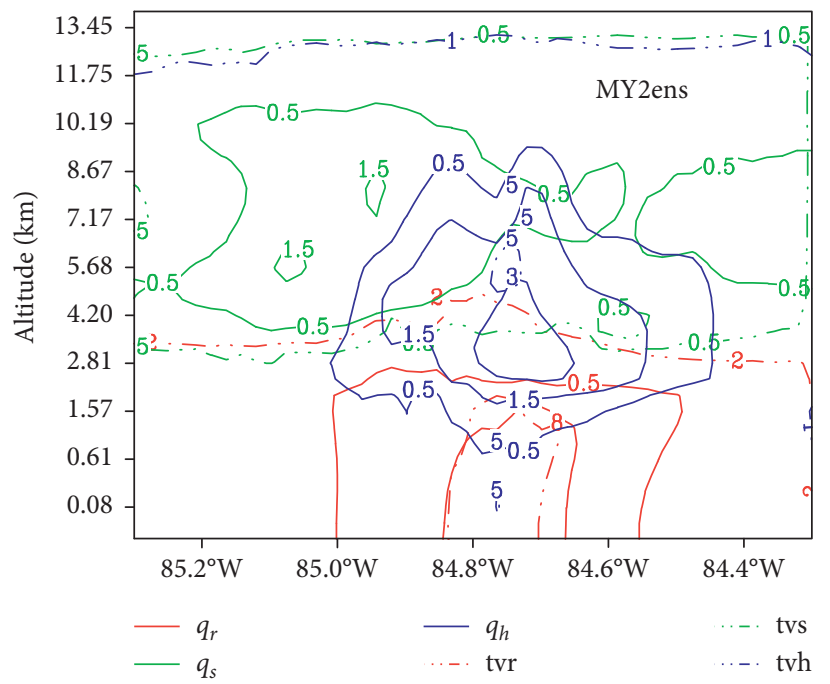

(c)

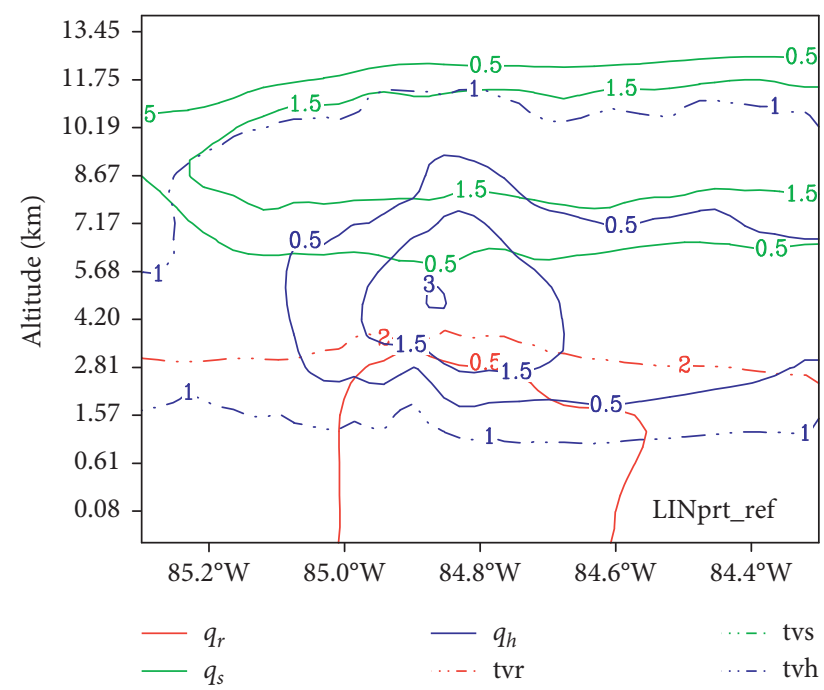

(b)

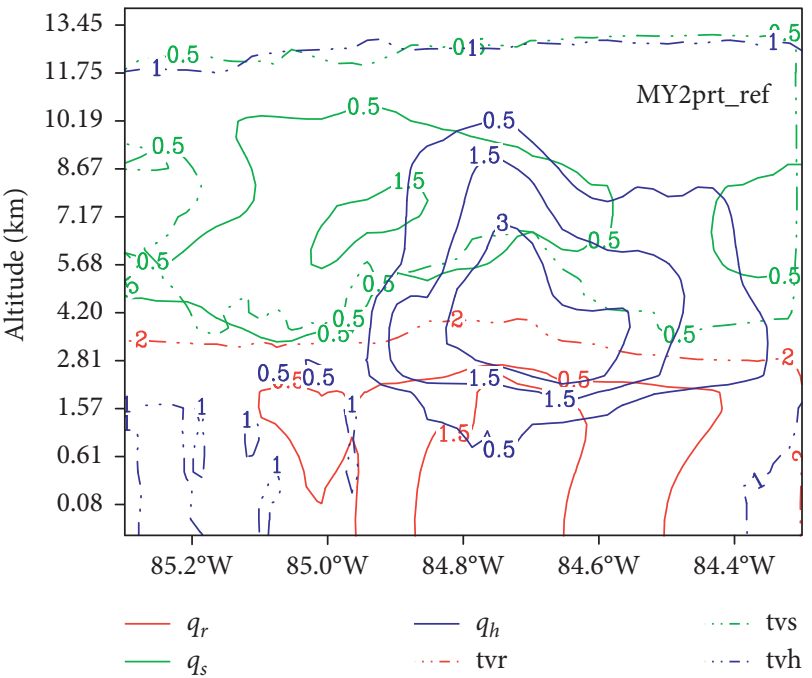

(d)

FiguRE 8: Vertical cross-sections of $q_{r}$ (red solid, $\mathrm{g} \cdot \mathrm{kg}^{-1}$ ), $q_{s}$ (green solid, $\mathrm{g} \cdot \mathrm{kg}^{-1}$ ), qh (blue solid, $\mathrm{g} \cdot \mathrm{kg}^{-1}$ ), and their corresponding terminal velocities (dot-dashed, $\mathrm{m} \cdot \mathrm{s}^{-1}$ ) at 0230 ZUTC along $34.4^{\circ} \mathrm{N}$ for (a) LINens, (b) LINprt_ref, (c) MY2ens, and (d) MY2prt_ref. Values shown in this figure are the meridional averages between $34.2^{\circ} \mathrm{N}$ and $34.6^{\circ} \mathrm{N}$.

forecasts capture the observed precipitation band oriented in the southwest-northeast direction at 01 UTC and two precipitation peaks at 02 UTC and 03 UTC, but there is clearly displacement in all forecasts. Although the precipitation distributions of members in LINprt_ref_K and LINens_K look similar, member 10 in LINprt_ref_K produces less spurious precipitation than member 9 in LINens_K. Further examination (not shown) indicates that, in member 10, negative perturbations (before resampling) prevail in the third forecast hour in northeastern Kansas, which results in lower terminal velocities in that area; thus, the spurious precipitation amount is smaller in the LINprt_ref_K member than that in LINens_K. The heavy rainfall areas (red areas in Figures 10(a), 10(c), 10(e), 10(g), $10(\mathrm{i})$, and $10(\mathrm{k})$ ) are also intuitively smaller in the LINprt_ref_K member, which is consistent with the result in
Figure 9(d) that the precipitation frequency of LINprt_ref_K is closer to the observations than that of LINens_K for precipitation greater than $10 \mathrm{~mm} \cdot \mathrm{h}^{-1}$. Since the Lin scheme often overestimates precipitation due to the unrealistically high terminal velocities, using stochastically perturbed but systematically smaller terminal velocities often helps precipitation forecasts.

Although the MY2 scheme members look similar throughout the forecast period, they differ in their details, especially at 03 UTC. At that time, the spurious precipitation area is smaller in member 9 in MY2prt_ref_K than in member 2 in MY2ens_K, which contributes to the smaller RMSE of the former than that of the latter (not shown). However, this better forecast occurs only in a few members, as shown in Figure 9(a). In most members in MY2prt_ref_K, the spurious precipitation areas are slightly larger than those 


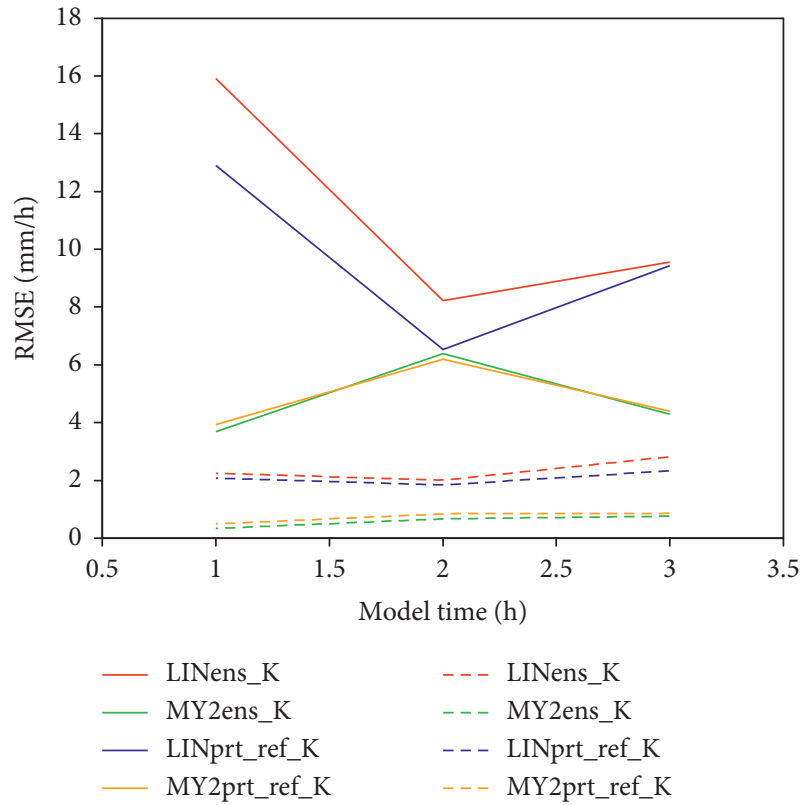

(a)

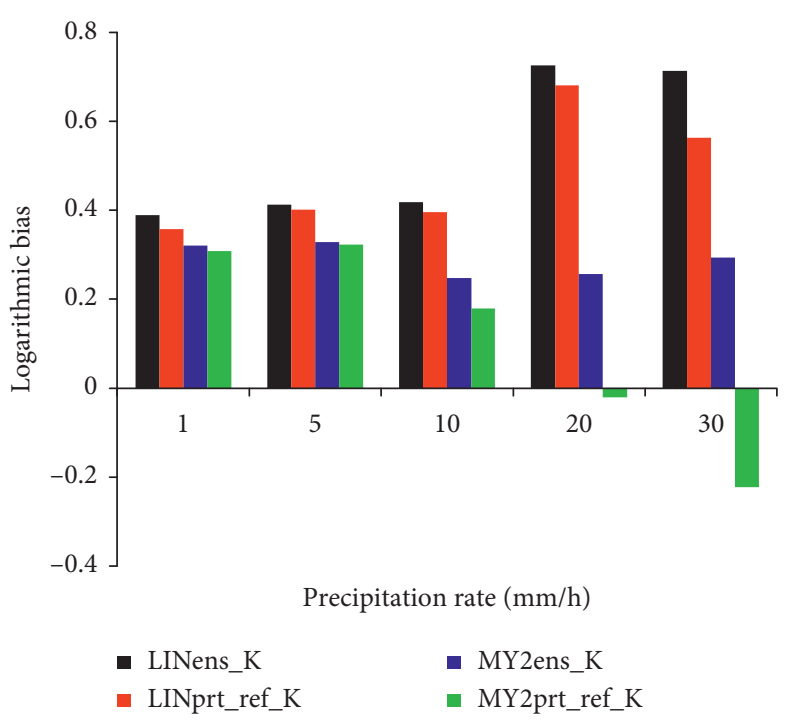

(c)

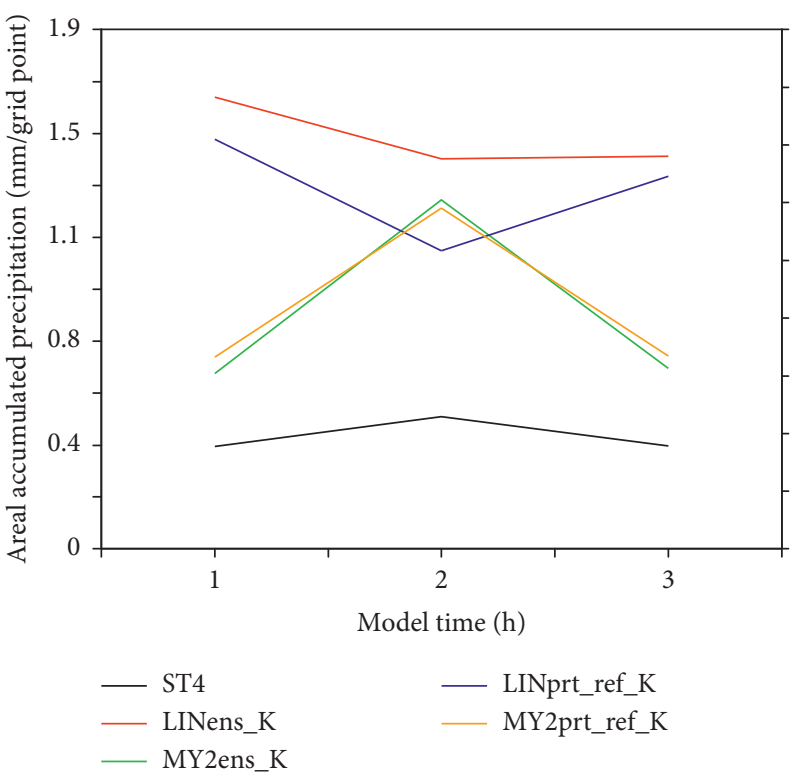

(b)

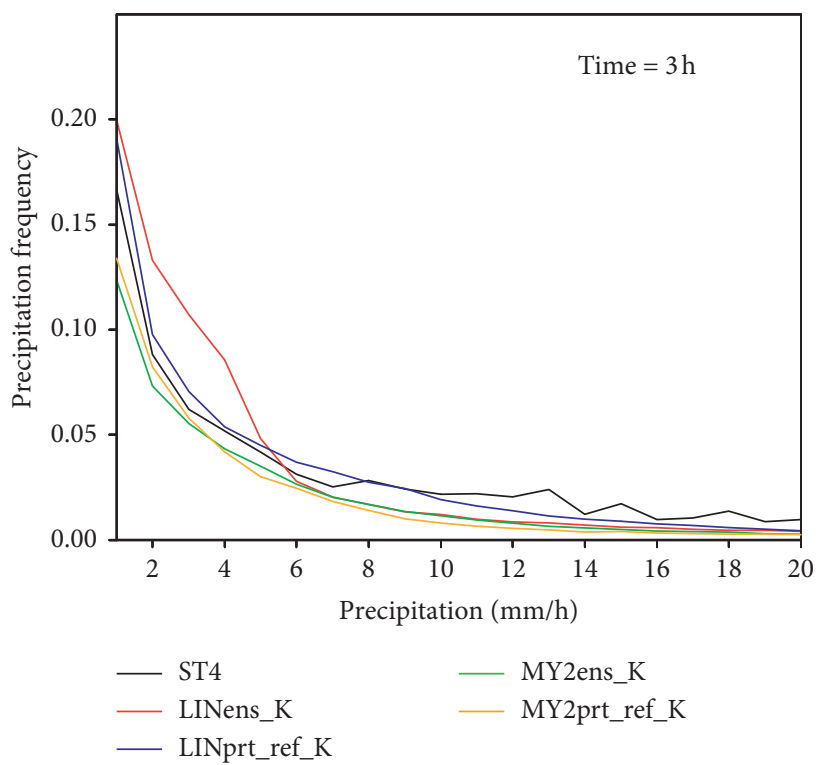

(d)

Figure 9: The same as in Figure 6, except for LINens_K, MY2ens_K, LINprt_ref, and MY2prt_ref_K.

in MY2ens_K at 03 UTC (not shown); this is the reason that the mean areal accumulated precipitation is slightly larger in MY2prt_ref_K than in MY2ens_K. Moreover, comparing Figures $9(\mathrm{~d})$ and $6(\mathrm{~d})$, symmetrically perturbing the terminal velocities in the MY2 scheme tends to produce more light rain $\left(<4 \mathrm{~mm} \cdot \mathrm{h}^{-1}\right)$, which implies that the symmetric perturbations tend to suppress the fall speeds of precipitation hydrometeors. This result is not contradictory to the fact that both MY2 scheme experiments overestimate the areal accumulated precipitation because these experiments produce larger precipitation areas than the observations. Additionally, the observed precipitation peaks at 02 UTC and 03 UTC (Figures 10(f), 10(h), 10(j), and 10(1)), especially for the south peak, are often underestimated by the MY2 scheme members. This result differhs from that in the Alabama case where precipitation is often overestimated, implying that symmetrically perturbing the terminal velocities may not help the precipitation prediction skill of the MY2 scheme when the precipitation peak is underestimated. 


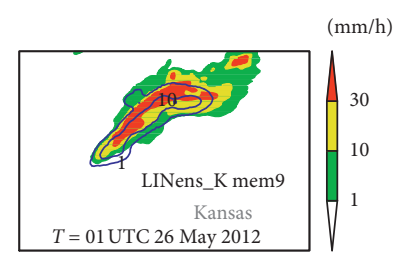

(a)

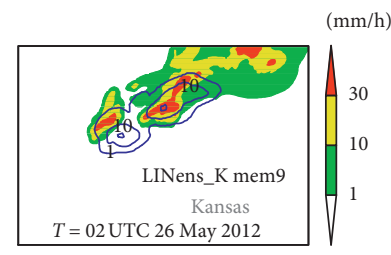

(e)

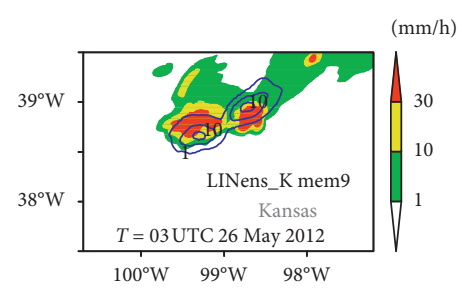

(i)

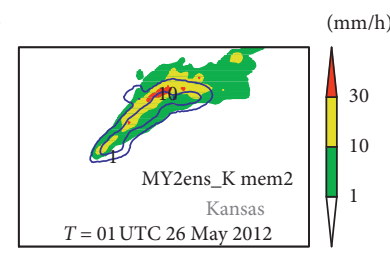

(b)

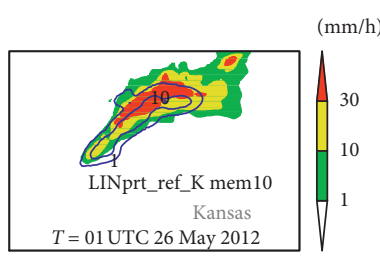

(c)

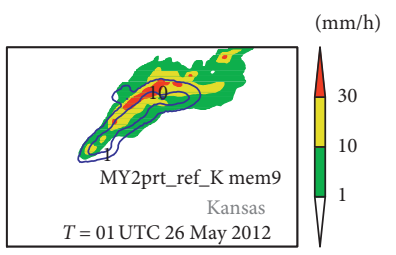

(d)

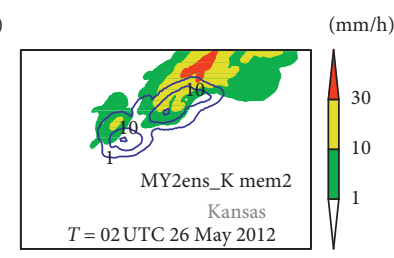

(f)

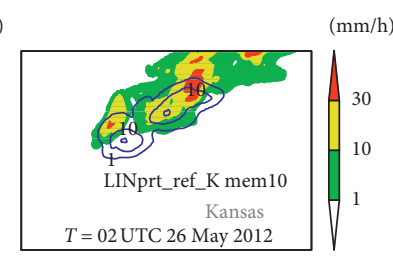

(g)

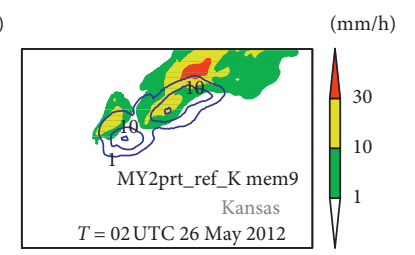

(h)

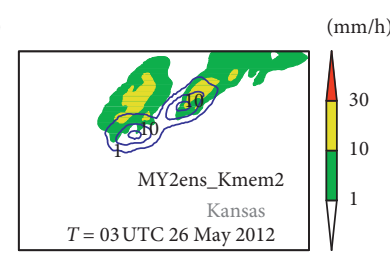

(j)

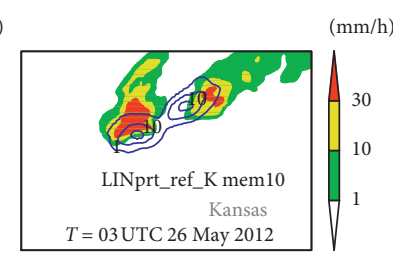

(k)

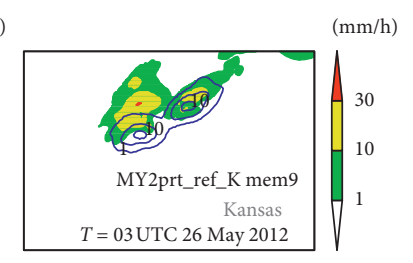

(1)

Figure 10: The same as in Figure 7, except for (a, e, i) LINens_K, (b, f, j) MY2ens_K, (c, g, k) LINprt_ref_K, and (d, h, l) MY2prt_ref_K.

\section{Summary and Conclusions}

The focus of this work was on the impact of stochastically perturbing the terminal velocities of hydrometeors on convective-scale ensemble forecasts of precipitation. An idealized supercell case was first employed. With this case and assuming that the MY2 scheme was the truth, the relationship between the precipitation and terminal velocities was investigated. After determining the perturbation amplitudes that minimized the terminal velocity difference between the Lin scheme and MY2 scheme, ensemble forecasts with stochastic perturbations of terminal velocities were performed and evaluated. Finally, the forecast skills of stochastically perturbing the terminal velocities were examined for both the one-moment and two-moment schemes with two real cases.

In terms of the results in Section 4, several conclusions were obtained: (i) compared to the MY2 scheme, the terminal velocities of snow and hail are approximately 3 times larger in the Lin scheme, which is an important source of the overestimated precipitation in the Lin scheme experiments; (ii) for the Lin scheme, it is better to perturb the terminal velocities using perturbations smaller than 1.0 , but note that these error characteristics depend on other parameters, such as the intercept parameters in the microphysics scheme; (iii) the Lin scheme often overestimates precipitation due to overestimating the terminal velocities; thus, symmetrically perturbing terminal velocities by $25 \%$ around their original values cannot effectively reduce the precipitation error when the Lin scheme is used; (iv) for the MY2 scheme, the use of symmetric terminal velocity perturbations within $\pm 25 \%$ reduces the precipitation error in the case when the MY2 scheme overestimates precipitation; this perturbation approach is ineffective in cases when precipitation is underpredicted.

As a preliminary work on terminal velocity perturbations, the potential benefits of using this type of perturbation were demonstrated. However, the experimental designs and results have many limitations; thus, it is not possible to establish a ranking of the configurations in terms of probabilistic forecast skill. First, two real cases are insufficient to confirm that terminal velocity perturbations can produce statistically significantly better performances than those without terminal velocity perturbations. Second, only a $3 \mathrm{~h}$ forecast was performed due to the computational limitation. Although the $3 \mathrm{~h}$ forecasts are sufficient for the scope of this work, the corresponding results are insufficient for longer-term forecasts, especially long-lived precipitation event forecasts. Longer forecast duration has to be considered for the comprehensive evaluation of the terminal velocity perturbations. In addition, the error characteristics of terminal velocities depend on many microphysical parameters, such as the intercept parameter and hydrometeor density; thus, the terminal velocity error characteristics obtained in this work may not be valid for other one-moment schemes. Finally, initial perturbations have not been considered but are essential in studies examining improvements in the probabilistic skill of this terminal velocity perturbation scheme. 


\section{Data Availability}

The Advanced Regional Prediction System (ARPS) can be downloaded at http://www.caps.ou.edu/ARPS/arpsdown. html, the GFS data are available at https://www.ncdc. noaa.gov/data-access/model-data/model-datasets/globalforcast-system-gfs, and the radar data can be downloaded at https://www.ncdc.noaa.gov/nexradinv/.

\section{Conflicts of Interest}

The authors declare that there are no conflicts of interest regarding the publication of this paper.

\section{Acknowledgments}

This work was jointly sponsored by the National Key Research and Development Program of China (2017YFC1502103), the National Natural Science Foundation of China (41505089, 41875129, 41505090, 41430427, and 41805070), the Startup Foundation for Introducing Talent of NUIST (2014R007), and the National Key Research and Development Program of China (2018YFC1506404).

\section{References}

[1] R. Buizza, J. Du, Z. Toth, and D. Hou, "Major operational ensemble prediction systems (EPS) and the future of EPS," Handbook of Hydrometeorological Ensemble Forecasting, Springer, Berlin, Germany, pp. 151-193, 2019.

[2] J. Berner, S.-Y. Ha, J. P. Hacker, A. Fournier, and C. Snyder, "Model uncertainty in a mesoscale ensemble prediction system: stochastic versus multiphysics representations," Monthly Weather Review, vol. 139, no. 6, pp. 1972-1995, 2011.

[3] H. M. Christensen, S.-J. Lock, I. M. Moroz, and T. N. Palmer, "Introducing independent patterns into the stochastically perturbed parametrization tendencies (SPPT) scheme," Quarterly Journal of the Royal Meteorological Society, vol. 143, no. 706, pp. 2168-2181, 2017.

[4] J. Berner, K. R. Fossell, S.-Y. Ha, J. P. Hacker, and C. Snyder, "Increasing the skill of probabilistic forecasts: understanding performance improvements from model-error representations," Monthly Weather Review, vol. 143, no. 4, pp. 12951320, 2015.

[5] I. Jankov, J. Beck, J. Wolff et al., "Stochastically perturbed parameterizations in an HRRR-based ensemble," Monthly Weather Review, vol. 147, no. 1, pp. 153-173, 2019.

[6] D. J. Stensrud, J.-W. Bao, and T. T. Warner, "Using initial condition and model physics perturbations in short-range ensemble simulations of mesoscale convective systems," Monthly Weather Review, vol. 128, no. 7, pp. 2077-2107, 2000.

[7] F. A. Eckel and C. F. Mass, "Aspects of effective mesoscale, short-range ensemble forecasting," Weather and Forecasting, vol. 20, no. 3, pp. 328-350, 2005.

[8] A. J. Clark, W. A. Gallus, and T.-C. Chen, "Contributions of mixed physics versus perturbed initial/lateral boundary conditions to ensemble-based precipitation forecast skill," Monthly Weather Review, vol. 136, no. 6, pp. 2140-2156, 2008.

[9] A. J. Clark, W. A. Gallus Jr., M. Xue, and F. Kong, "A comparison of precipitation forecast skill between small convection-allowing and large convection-parameterizing ensembles," Weather and Forecasting, vol. 24, no. 4, pp. 1121-1140, 2009.
[10] A. J. Clark, W. A. Gallus Jr., M. Xue, and F. Kong, "Convection-allowing and convection-parameterizing ensemble forecasts of a mesoscale convective vortex and associated severe weather environment," Weather and Forecasting, vol. 25, no. 4, pp. 1052-1081, 2010.

[11] J. P. Hacker, S.-Y. Ha, C. Snyder et al., "The U.S. air forceweather agency's mesoscale ensemble: scientific description and performance results," Tellus A: Dynamic Meteorology and Oceanography, vol. 63, no. 3, pp. 625-641, 2011.

[12] Y. Wang, M. Bellus, C. Wittmann et al., "The Central European limited-area ensemble forecasting system: aladinLAEF," Quarterly Journal of the Royal Meteorological Society, vol. 137, no. 655, pp. 483-502, 2011.

[13] C. Melhauser, F. Zhang, Y. Weng, Y. Jin, H. Jin, and Q. Zhao, "A multiple-model convection-permitting ensemble examination of the probabilistic prediction of tropical cyclones: hurricanes sandy (2012) and edouard (2014)," Weather and Forecasting, vol. 32, no. 2, pp. 665-688, 2017.

[14] A. J. Clark, I. L. Jirak, S. R. Dembek et al., "The community leveraged unified ensemble (CLUE) in the $2016 \mathrm{NOAA} /$ hazardous weather testbed spring forecasting experiment," Bulletin of the American Meteorological Society, vol. 99, no. 7, pp. 1433-1448, 2018.

[15] I. Jankov, J. Berner, J. Beck et al., "A performance comparison between multiphysics and stochastic approaches within a north American RAP ensemble," Monthly Weather Review, vol. 145, no. 4, pp. 1161-1179, 2017.

[16] G. S. Romine, C. S. Schwartz, J. Berner et al., "Representing forecast error in a convection-permitting ensemble system," Monthly Weather Review, vol. 142, no. 12, pp. 4519-4541, 2014.

[17] C. Wastl, Y. Wang, A. Atencia, and C. Wittmann, "Independent perturbations for physics parametrization tendencies in a convection-permitting ensemble (pSPPT)," Geoscientific Model Development, vol. 12, no. 1, pp. 261-273, 2019.

[18] P. Ollinaho, S.-J. Lock, M. Leutbecher et al., "Towards process-level representation of model uncertainties: stochastically perturbed parametrizations in the ECMWF ensemble," Quarterly Journal of the Royal Meteorological Society, vol. 143, no. 702, pp. 408-422, 2017.

[19] R. Buizza, M. Milleer, and T. N. Palmer, "Stochastic representation of model uncertainties in the ECMWF ensemble prediction system," Quarterly Journal of the Royal Meteorological Society, vol. 125, no. 560, pp. 2887-2908, 1999.

[20] T. N. Palmer, R. Buizza, F. Doblas-Reyes et al., "Stochastic parametrization and model uncertainty," , p. 42, 2009 ECMWF Technical Memoranda Technical memorandum 598.

[21] F. Bouttier, B. Vié, O. Nuissier, and L. Raynaud, "Impact of stochastic physics in a convection-permitting ensemble," Monthly Weather Review, vol. 140, no. 11, pp. 3706-3721, 2012.

[22] S. Fresnay, A. Hally, C. Garnaud, E. Richard, and D. Lambert, "Heavy precipitation events in the Mediterranean: sensitivity to cloud physics parameterisation uncertainties," Natural Hazards and Earth System Sciences, vol. 12, no. 8, pp. 26712688, 2012.

[23] A. Hally, E. Richard, S. Fresnay, and D. Lambert, "Ensemble simulations with perturbed physical parametrizations: preHyMeX case studies," Quarterly Journal of the Royal Meteorological Society, vol. 140, no. 683, pp. 1900-1916, 2014.

[24] H. M. Christensen, I. M. Moroz, and T. N. Palmer, "Stochastic and perturbed parameter representations of model uncertainty in convection parameterization," Journal of the Atmospheric Sciences, vol. 72, no. 6, pp. 2525-2544, 2015. 
[25] X. Qiao, S. Wang, and J. Min, “The impact of a stochastically perturbing microphysics scheme on an idealized supercell storm," Monthly Weather Review, vol. 146, no. 1, pp. 95-118, 2018.

[26] C. Sanchez, K. D. Williams, and M. Collins, "Improved stochastic physics schemes for global weather and climate models," Quarterly Journal of the Royal Meteorological Society, vol. 142, no. 694, pp. 147-159, 2016.

[27] G. Shutts and A. C. Pallarès, "Assessing parametrization uncertainty associated with horizontal resolution in numerical weather prediction models," Philosophical Transactions of the Royal Society A: Mathematical, Physical and Engineering Sciences, vol. 372, no. 2018, Article ID 20130284, 2014.

[28] S. Wang, X. Qiao, J. Min, and X. Zhuang, "The impact of stochastically perturbed parameterizations on tornadic supercell cases in east China," Monthly Weather Review, vol. 147 , no. 1, pp. 199-220, 2019.

[29] M. S. Gilmore, J. M. Straka, and E. N. Rasmussen, "Precipitation uncertainty due to variations in precipitation particle parameters within a simple microphysics scheme," Monthly Weather Review, vol. 132, no. 11, pp. 2610-2627, 2004.

[30] H. Morrison, G. Thompson, and V. Tatarskii, "Impact of cloud microphysics on the development of trailing stratiform precipitation in a simulated squall line: comparison of oneand two-moment schemes," Monthly Weather Review, vol. 137, no. 3, pp. 991-1007, 2009.

[31] D. T. Dawson, M. Xue, J. A. Milbrandt, and M. K. Yau, "Comparison of evaporation and cold pool development between single-moment and multimoment bulk microphysics schemes in idealized simulations of tornadic thunderstorms," Monthly Weather Review, vol. 138, no. 4, pp. 1152-1171, 2010.

[32] H. Morrison and J. Milbrandt, "Comparison of two-moment bulk microphysics schemes in idealized supercell thunderstorm simulations," Monthly Weather Review, vol. 139, no. 4, pp. 1103-1130, 2011.

[33] A. Parodi and K. Emanuel, "A theory for buoyancy and velocity scales in deep moist convection," Journal of the Atmospheric Sciences, vol. 66, no. 11, pp. 3449-3463, 2009.

[34] M. S. Singh and P. A. O'Gorman, "Influence of microphysics on the scaling of precipitation extremes with temperature," Geophysical Research Letters, vol. 41, no. 16, pp. 6037-6044, 2014.

[35] G. H. Bryan and H. Morrison, "Sensitivity of a simulated squall line to horizontal resolution and parameterization of microphysics," Monthly Weather Review, vol. 140, no. 1, pp. 202-225, 2012.

[36] A. Morales, H. Morrison, and D. J. Posselt, "Orographic precipitation response to microphysical parameter perturbations for idealized moist nearly neutral flow," Journal of the Atmospheric Sciences, vol. 75, no. 6, pp. 1933-1953, 2018.

[37] S. E. Yuter, D. E. Kingsmill, L. B. Nance, and M. Löffler-Mang, "Observations of precipitation size and fall speed characteristics within coexisting rain and wet snow," Journal of Applied Meteorology and Climatology, vol. 45, no. 10, pp. 1450-1464, 2006.

[38] Y.-L. Lin, R. D. Farley, and H. D. Orville, "Bulk parameterization of the snow field in a cloud model," Journal of Climate and Applied Meteorology, vol. 22, no. 6, pp. 1065-1092, 1983.

[39] S.-Y. Hong, J. Dudhia, and S.-H. Chen, "A revised approach to ice microphysical processes for the bulk parameterization of clouds and precipitation," Monthly Weather Review, vol. 132, no. 1, pp. 103-120, 2004.

[40] G. Thompson, R. M. Rasmussen, and K. Manning, "Explicit forecasts of winter precipitation using an improved bulk microphysics scheme. Part I: description and sensitivity analysis," Monthly Weather Review, vol. 132, no. 2, pp. 519$542,2004$.

[41] J. A. Milbrandt and M. K. Yau, "A multimoment bulk microphysics parameterization. Part I: analysis of the role of the spectral shape parameter," Journal of the Atmospheric Sciences, vol. 62, no. 9, pp. 3051-3064, 2005.

[42] H. Morrison, J. A. Curry, and V. I. Khvorostyanov, "A new double-moment microphysics parameterization for application in cloud and climate models. Part I: description," Journal of the Atmospheric Sciences, vol. 62, no. 6, pp. 1665-1677, 2005.

[43] T. B. Low and R. List, "Collision, coalescence and breakup of raindrops. Part I: experimentally established coalescence efficiencies and fragment size distributions in breakup," Journal of the Atmospheric Sciences, vol. 39, no. 7, pp. 1591-1606, 1982.

[44] A. L. Igel, M. R. Igel, and S. C. van den Heever, "Make it a double? Sobering results from simulations using single-moment microphysics schemes," Journal of the Atmospheric Sciences, vol. 72, no. 2, pp. 910-925, 2015.

[45] G. Montero-Martínez, A. B. Kostinski, R. A. Shaw, and F. García-García, "Do all raindrops fall at terminal speed?" Geophysical Research Letters, vol. 36, no. 11, Article ID L11818, 2009.

[46] X. Zhang, "Multiscale characteristics of different-source perturbations and their interactions for convection-permitting ensemble forecasting during SCMREX," Monthly Weather Review, vol. 147, no. 1, pp. 291-310, 2019.

[47] M. Xue, K. K. Droegemeier, and V. Wong, "The advanced regional prediction system (ARPS) - a multi-scale nonhydrostatic atmospheric simulation and prediction model. Part I: model dynamics and verification," Meteorology and Atmospheric Physics, vol. 75, no. 3-4, pp. 161-193, 2000.

[48] M. Xue, K. K. Droegemeier, V. Wong et al., "The advanced regional prediction system (ARPS) - a multi-scale nonhydrostatic atmospheric simulation and prediction tool. Part II: model physics and applications," Meteorology and Atmospheric Physics, vol. 76, no. 1-4, pp. 143-165, 2001.

[49] C. Sheng, S. Gao, and M. Xue, "Short-range prediction of a heavy precipitation event by assimilating Chinese CINRADSA radar reflectivity data using complex cloud analysis," Meteorology and Atmospheric Physics, vol. 94, no. 1-4, pp. 167-183, 2006.

[50] R. S. Schumacher, A. J. Clark, M. Xue, and F. Kong, "Factors influencing the development and maintenance of nocturnal heavy-rain-producing convective systems in a storm-scale ensemble," Monthly Weather Review, vol. 141, no. 8, pp. 2778-2801, 2013.

[51] Y. Pan, M. Xue, and G. Ge, "Incorporating diagnosed intercept parameters and the graupel category within the ARPS cloud analysis system for the initialization of double-moment microphysics: testing with a squall line over South China," Monthly Weather Review, vol. 144, no. 1, pp. 371-392, 2016.

[52] N. Snook, Y. Jung, J. Brotzge, B. Putnam, and M. Xue, "Prediction and ensemble forecast verification of hail in the supercell storms of 20 may 2013," Weather and Forecasting, vol. 31, no. 3, pp. 811-825, 2016.

[53] G. Wang, D. Wang, J. Yang, and L. Liu, "Evaluation and correction of quantitative precipitation forecast by stormscale NWP model in jiangsu, China," Advances in Meteorology, vol. 2016, pp. 1-13, 2016.

[54] X. Qiao, S. Wang, and J. Min, “A stochastic perturbed parameterization tendency scheme for diffusion (SPPTD) and 
its application to an idealized supercell simulation," Monthly Weather Review, vol. 145, no. 6, pp. 2119-2139, 2017.

[55] J. Gao and D. J. Stensrud, "Assimilation of reflectivity data in a convective-scale, cycled 3DVAR framework with hydrometeor classification," Journal of the Atmospheric Sciences, vol. 69, no. 3, pp. 1054-1065, 2012.

[56] C. S. Schwartz, G. S. Romine, M. L. Weisman et al., "A realtime convection-allowing ensemble prediction system initialized by mesoscale ensemble kalman filter analyses," Weather and Forecasting, vol. 30, no. 5, pp. 1158-1181, 2015.

[57] Y. Lin and K. E. Mitchell, "The NCEP stage II/IV hourly precipitation analyses: development and applications," in Proceedings of the 19th Conference on Hydrology, American Meteor Society, San Diego, CA, USA, January 2005, http:// ams.confex.com/ams/pdfpapers/83847.pdf.

[58] B. J. Putnam, M. Xue, Y. Jung, N. Snook, and G. Zhang, "The analysis and prediction of microphysical states and polarimetric radar variables in a mesoscale convective system using double-moment microphysics, multinetwork radar data, and the ensemble kalman filter," Monthly Weather Review, vol. 142, no. 1, pp. 141-162, 2014.

[59] J. Gao, M. Xue, K. Brewster, and K. K. Droegemeier, "A threedimensional variational data analysis method with recursive filter for Doppler radars," Journal of Atmospheric and Oceanic Technology, vol. 21, no. 3, pp. 457-469, 2004.

[60] M.-D. Chou, "A solar radiation model for use in climate studies," Journal of the Atmospheric Sciences, vol. 49, no. 9, pp. 762-772, 1992.

[61] M.-D. Chou and M. J. Suarez, "An efficient thermal infrared radiation parameterization for use in general circulation models," NASA, Washington, DC, USA, Technical Memorandum No. 104606, 1994.

[62] W.-Y. Sun and C.-Z. Chang, "Diffusion model for a convective layer. Part I: numerical simulation of convective boundary layer," Journal of Climate and Applied Meteorology, vol. 25, no. 10, pp. 1445-1453, 1986.

[63] C.-H. Moeng, "A large-eddy-simulation model for the study of planetary boundary-layer turbulence," Journal of the Atmospheric Sciences, vol. 41, no. 13, pp. 2052-2062, 1984.

[64] N. Snook and M. Xue, "Effects of microphysical drop size distribution on tornadogenesis in supercell thunderstorms," Geophysical Research Letters, vol. 35, no. 24, pp. 851-854, 2008.

[65] L. Wu and G. W. Petty, "Intercomparison of bulk microphysics schemes in model simulations of polar lows," Monthly Weather Review, vol. 138, no. 6, pp. 2211-2228, 2010.

[66] K. Van Weverberg, A. M. Vogelmann, W. Lin et al., "The role of cloud microphysics parameterization in the simulation of mesoscale convective system clouds and precipitation in the tropical western Pacific," Journal of the Atmospheric Sciences, vol. 70, no. 4, pp. 1104-1128, 2013.

[67] L. P. D'Adderio, F. Porcù, and A. Tokay, "Identification and analysis of collisional breakup in natural rain," Journal of the Atmospheric Sciences, vol. 72, no. 9, pp. 3404-3416, 2015. 